\title{
Interaction between an eddy and a zonal jet
}

\section{Part I. One-and-a-half-layer model}

\author{
Frédéric O. Vandermeirsch ${ }^{\mathrm{a}, \mathrm{b}}$, Xavier J. Carton ${ }^{\mathrm{a}, \mathrm{b}},{ }^{*}$ and Yves G. Morel ${ }^{\mathrm{a}}$
}

\begin{abstract}
a : Service Hydrographique et Océanographique de la Marine, Centre Militaire d'Océanographie, Brest, France b : Institut Francais de Recherche et d'Exploitation de la MER, Laboratoire de Physique des Océans, Brest, France
\end{abstract}

*: Corresponding author : Tel.:+33-2-9822-4915; fax:+33-2-9822-4496.

\begin{abstract}
:
The interaction between a stable zonal jet and a vortex is studied numerically with two one-and-a-half layer models, one with quasi-geostrophic dynamics, the other with shallow-water equations. In both models, simulations on the f-plane evidence three regimes occuring with increasing vortex strength: (regime 1) weak vortices do not cross the jet and steadily drift along it; (regime 2) stronger vortices cross the jet, tear an opposite-sign meander from the jet with which they pair as a dipole; the trajectory of this dipole depends on the strength of the initial vortex; since most dipoles are asymmetric, they veer back towards the jet axis where they are split apart in the ambient shear; (regime 3) even stronger vortices cross the jet and tear a vorticity filament without dipole formation.

The influence of various physical parameters on jet-vortex interaction is studied. In particular, $\beta$-effect is not sufficient to drive all vortices through the jet.

Numerical simulations show that jet crossing occurs when the maximum velocity of the vortex is larger than, and opposite to, that of the jet. This allows the mathematical derivation of an analytical criterion for jet crossing in both models, which relates the potential vorticity anomalies of the jet and vortex, the vortex and internal deformation radii. In the shallow-water model, an asymmetry is observed between anticyclones north of the jet and cyclones south of it. The role of a spatially varying deformation radius and of vortex cyclostrophy on this asymmetry is discussed.
\end{abstract}

Keywords: Rotating fluids; Stratified flow; Nonlinear equations; Jets; Vortices; Potential vorticity 


\section{Review of previous work and outline}

Jets and coherent vortices play an essential role in mesoscale ocean dynamics, by carrying a large amount of heat and momentum. Jets and vortices can interact in several manners:

- unstable jets often form vortices (e.g. the Gulf-Stream rings) which can later distort their parent jet. Indeed, Gulf-Stream rings either drift away from the jet and slowly die by ventilation or mixing, or they interact again with the Gulf-Stream again and may be absorbed (Richardson, 1980).

- another interaction is that occuring between a strong oceanic jet (the Azores Current, for example) and vortices formed elsewhere (in that instance, meddies generated on the Iberian continental slope). Such an interaction produces large meanders on the zonal jet (Käse \& Zenk, 1996; Tychensky \& Carton, 1998). When the meddy has crossed the jet, a southward meander finally occludes and produces a surfaceintensified cyclone which pairs with the meddy as a baroclinic dipole (Richardson \& Tychensky, 1998).

Thus, the interaction of a zonal jet with a coherent vortex is of interest for ocean dynamics. Therefore it has been the subject of many process studies using contour dynamics:

- in a one-and-a-half layer quasi-geostrophic framework, Stern and Flierl (1987) showed that the presence of a point-vortex can induce a meander on a stable jet; this meander has a strength (area-integrated potential vorticity) equal and opposite to that of the point-vortex (an analytical result in the limit of weak eddy-jet interaction). This interaction was simulated numerically beyond the linear stage, but due to the lack of contour surgery, the point vortex could not cross the vorticity front.

- in a two-dimensional framework, Bell (1990) derived analytically the formulae for the deformation of the potential vorticity front and for the motion of a point vortex, again in the limit of weak eddy-jet interaction. The meridional position and velocity of the vortex were related to the jet velocity and deformation, in particular by means of integral invariants and via asymptotic expansions.

- Stern (1991) revisited this problem numerically in the barotropic model, now with a finite-area vortex. He showed that the vortex could be entrained into the shear flow region, a process accompanied by large filament formation and winding. He defined an entrainment velocity by dividing the area of fluid entrained in a fixed time by the characteristic length scale of the problem; this velocity is proportional to the square root of the vortex circulation and to the shear flow intensity.

- in both barotropic and one-and-a-half layer quasi-geostrophic cases, Bell and Pratt (1992) showed that a vortex could trigger strong perturbations on an unstable jet. Such perturbations can evolve nonlinearly into dipoles which drift away from the jet. In the case where the eddy rotation was opposite to the jet direction, a stagnation point was noted in the vicinity of the vorticity front. 
- In the absence of an external eddy, the propagation and amplification of an initial wave on a potential vorticity front has been thoroughly studied by Stern (1985), Pratt and Stern (1986) and Pratt (1988) for a single active layer. A formula for the frontal deviation with respect to time and longitude was derived for several cases (small amplitude periodic disturbances, long wave limit) and in particular for disturbances of finite amplitude and small curvature. The nonlinear meandering and eddy pinching off processes were analyzed by means of this equation. In the special case of a fluid intrusion along a coast, a vorticity front can also exhibit lee wave formation and wave breaking (Stern and Pratt, $1985)$.

Other process studies were conducted in stratified flows; they will be recalled in part II of this paper.

Here, the interaction between a finite-area vortex and a stable zonal jet is studied in a one-and-a-half layer model, first with quasi-geostrophic dynamics, then with shallowwater equations. This latter framework is a novelty compared with former studies and seems necessary to take into account large isopycnal deviations associated with fronts and eddies in the ocean. The present study focuses on the nonlinear evolutions of the flow, for which linearized equations and perturbation expansions are not applicable. Several questions are raised by observations and are unanswered by previous studies: - What are the nonlinear evolutions of the jet and vortex when their respective intensities and sizes are varied?

- If the vortex crosses the jet, will perturbations with characteristic wavelengths develop on the latter?

- Can the crossing of the jet by the vortex be analytically predicted ?

- Is $\beta$-effect a limiting or favoring factor of jet crossing ?

- More generally, do quasi-geostrophic and shallow-water dynamics exhibit similarities or noticeable differences in modeling this interaction?

- In particular, does the shallow-water model exhibit cyclone-anticyclone asymmetry for jet-vortex interaction as it does for vortex merger (Valcke \& Verron, 1996) ?

- If so, where does this asymmetry come from ?

These questions motivate the present process study.

After recalling the model equations and numerics (section 2), we determine the various nonlinear evolutions of the jet and vortex in the quasi-geostrophic model (section 3); we also derive physically an analytical criterion for jet crossing, and test our results for sensitivity to parameter variations. In section 4 , the generalization of this study to shallow-water dynamics is presented, with emphasis on the influence of velocity and vorticity fields on cyclone-anticyclone asymmetry. Conclusions are given in section 5 . 


\section{$2 \quad$ Physical and numerical models}

\subsection{The shallow-water (SW) model}

In the absence of forcing and of dissipation, the shallow-water equations are written in a one-and-a-half layer model:

$$
\begin{gathered}
{\left[\partial_{t}+u \partial_{x}+v \partial_{y}\right] u-f v=-g^{\prime} \partial_{x} h} \\
{\left[\partial_{t}+u \partial_{x}+v \partial_{y}\right] v+f u=-g^{\prime} \partial_{y} h} \\
{\left[\partial_{t}+u \partial_{x}+v \partial_{y}\right] h+h\left[\partial_{x} u+\partial_{y} v\right]=0}
\end{gathered}
$$

where $u, v$ and $h$ are respectively the two components of the horizontal velocity and the thickness of the active ocean layer. The reduced gravity is $g^{\prime}=g \Delta \rho / \rho$, where $\rho$ is the active layer density and $\Delta \rho<<\rho$ is the density jump between layers. The planetary vorticity is $f=f_{0}+\beta y$ and the relative vorticity is $\zeta=\partial_{x} v-\partial_{y} u$.

These equations ensure conservation of potential vorticity:

$$
q=\frac{\zeta+f}{h}
$$

Jet and vortex dynamics are usually described in term of potential vorticity anomaly (hereafter PVA), computed from a state of rest:

$$
\delta q=\frac{\zeta+f}{h}-\frac{f_{0}}{H}=\frac{\zeta-f_{0} \eta / H+\beta y}{H+\eta}
$$

where $H$ is the thickness of the active layer at rest, and $\eta=h-H$ is the local variation of layer thickness.

\subsection{The quasi-geostrophic (QG) model}

The quasi-geostrophic model is the limit of the shallow-water equations for weak flows (small Rossby number $R o=U / f_{0} L$ ), for comparable Coriolis and buoyancy effects (Burger number $B u=g^{\prime} H / f_{0}^{2} L^{2}$ of order unity), and for small meridional variation of Coriolis parameter ( $\beta$-plane approximation). The first two hypotheses imply that the interface elevation remains weak compared with the active layer thickness.

In the quasi-geostrophic model, the horizontal velocity derives from a streamfunction $u=-\partial_{y} \psi, v=\partial_{x} \psi$, with $\psi=g^{\prime} h / f_{0}$, and in the absence of forcing and dissipation, potential vorticity is conserved:

$$
\left[\partial_{t}+u \partial_{x}+v \partial_{y}\right] Q=\left[\partial_{t}+J(\psi, \cdot)\right] Q=0
$$

where $J(a, b)$ is the Jacobian operator. Quasi-geostrophic potential vorticity is the analogue of shallow-water PV for small Ro and order one $B u$ :

$$
Q=\nabla^{2} \psi-\gamma^{2} \psi+f
$$

for the one-and-a-half layer model, with $\gamma^{2}=1 / R_{d}^{2}=f_{0}^{2} / g^{\prime} H . \quad R_{d}$ is the internal radius of deformation. 


\subsection{Initial conditions}

Initial conditions for both models are a zonal jet composed of two strips of constant PVA and a circular vortex composed of a disk of constant PVA (see Figure 1). These PVA add up in the initial conditions, but the velocity fields do so only in the quasigeostrophic model, which has a linear relation between PVA and velocity.

In the quasi-geostrophic model, the vortex is anticyclonic (considering the symmetry of the equations) and it is initially located north of the jet axis at $y=y_{T}$. The PVA distribution of the jet $\delta Q_{J}(y)$ and that of the vortex $\delta Q_{T}(y)$ are inverted to calculate the velocity field:

$$
\begin{gathered}
\delta Q_{J}(y)=\left\{\begin{array}{r}
\Delta Q_{J} / 2, y>0 \\
-\Delta Q_{J} / 2, y<0
\end{array} \Longrightarrow u_{J}(y)=\Delta Q_{J} /(2 \gamma) \exp (-\gamma|y|)\right. \\
\delta Q_{T}(r)=\left\{\begin{array}{c}
\Delta Q_{T},|r|<R_{T} \\
0,|r|>R_{T}
\end{array} \Longrightarrow v_{\theta, T}(r)=\left\{\begin{array}{c}
\Delta Q_{T} R_{T} K_{1}\left(\gamma R_{T}\right) I_{1}(\gamma r) \\
\Delta Q_{T} R_{T} I_{1}\left(\gamma R_{T}\right) K_{1}(\gamma r)
\end{array}\right.\right.
\end{gathered}
$$

where $\Delta Q_{J}$ and $\Delta Q_{T}$ are the jumps in PVA across the jet and vortex boundaries; $R_{T}$ is the vortex radius; $I_{1}$ and $K_{1}$ are the modified first-order Bessel functions of the first and of the second kind, respectively. Both vortex and jet are unconditionally stable by virtue of the Rayleigh-Kuo's theorem (in the quasi-geostrophic model).

The jet and vortex are also initialized with piecewise-constant PVA $\delta q$ in the shallowwater model, so that:

$$
\zeta-f_{0} \eta / H=\delta q(H+\eta) / H
$$

In this model, the vortex can be an anticyclone initially north of the jet or a cyclone south of it. The pressure (or height) field is computed via the gradient-wind balance equation (the velocity divergence equation truncated at second order in Rossby number):

$$
-g^{\prime} \nabla^{2} \eta=f_{0} \zeta+2 J(u, v)
$$

These two equations form a relation between $\eta, u, v$ which is solved iteratively. Though it is not an exact equilibrium of the shallow-water equations, this relation minimizes the initial generation of gravity waves, and it has already been used in numerical models (e.g. Valcke and Verron, 1996).

\subsection{Numerical implementation}

Jet-vortex interaction is studied here in a zonal channel. A contour surgery model would be best suited to reproduce potential vorticity fronts (see Dritschel \& Saravanan, 1994 for instance). But such models are not yet available with prescribed inflow and radiative outflow conditions which are needed to avoid spurious interaction of unstable waves via zonal periodicity. Therefore we use finite-difference versions of the quasi-geostrophic and shallow-water models, which possess inflow/outflow conditions. Such models have been used widely to simulate large and meso-scale ocean dynamics (Holland, 1978; Bleck \& Boudra, 1986). Finite-difference code imply an initial smoothing of potential vorticity fronts to avoid Gibbs' numerical instability 
(Valcke, 1995). This smoothing is achieved via a four-point filter and by the finite mesh of the grid; it is limited and does not physically alter the evolution of the flow. The computational domain is thus a zonal channel with free-slip conditions at the northern and southern walls. An inflow is imposed on the western side, while the eastern boundary condition is radiative (Orlanski, 1976). The domain size is discretized over $151 \times 151$ nodes in the quasi-geostrophic model; the shallow-water model domain has $151 \times 171$ points, with a sponge layer to absorb outgoing flow. Horizontal resolution has been increased to $251 \times 251$ and $251 \times 281$ points respectively, for a detailed inspection of jet-vortex interaction. The time-step is computed from the CFL stability condition. In the shallow-water model, the variables are computed on an Arakawa C-grid.

All model parameters are rendered dimensionless with the vortex radius $R_{T}=1$, with a unit potential vorticity jump across the jet $\Delta Q_{J}=1$; the layer thickness is $H=1$. Biharmonic friction is used in the numerical models to remove enstrophy accumulation at small scales: a term $-\nu \nabla^{4} u, v$ is added to the right-hand side of momentum equations in system (1), and a term $-\nu \nabla^{6} \psi$ to the right-hand side of equation (2). Since the potential vorticity gradients are not infinite, no spurious overshooting occurs when using biharmonic dissipation. At the lowest resolution $(\Delta x=\Delta y=0.125)$, the computational domain length is $\left.L_{x}=18.75\right)$, the dimensionless viscosity is $\nu=310^{-6}$. We have checked that physical results do not vary qualitatively between low and high-resolution simulations.

First, these models are tested for the present study: can the steep vorticity gradients in the initial conditions and the radiative boundary conditions remain numerically stable and accurate for long durations ? Sinusoidal perturbations with wavelength $2 \pi / k$ are added initially to the potential vorticity front, in the absence of the vortex. Theory shows that these waves have a phase speed:

$$
c_{r}(k)=u_{0}-\frac{\Delta Q_{J}}{2 \sqrt{k^{2}+\gamma^{2}}}, \quad u_{0}=\Delta Q_{J} / 2 \gamma
$$

The accuracy of the phase speed is verified numerically with a Hovemuller (latitude/time) diagram (not shown). Moreover, the waves are advected out of the domain without reflection at the eastern boundary.

\section{Eddy interaction with a zonal jet in the quasi- geostrophic model}

This section is devoted to the description and classification of the various nonlinear regimes of jet-vortex interaction on the $f$-plane (subsection 1 ), using numerical experiments with a quasi-geostrophic model. These results lead to an analytical criterion giving the condition on vortex size and strength for jet crossing (subsection 2). Finally (subsection 3 ), the sensitivity of this interaction to several physical parameters (including $\beta$-effect) is investigated. 


\subsection{Nonlinear regimes of jet-vortex interaction}

The initial conditions of the numerical model are chosen as an anticyclonic vortex located north of an eastward jet on the $f$-plane; the case of a cyclone north of the jet will also be mentioned. All other cases (vortex south of the jet and/or westward jet) can be deduced from these two cases by symmetry of the quasi-geostrophic equations. The anticyclone trajectories are studied with respect to its intensity $\Delta Q_{T}$ while the other parameters are kept fixed, with values characteristic of mid-latitude vortices (see table 1 ), though $\beta=0$. Figure 2 a presents the schematic diagram of the three nonlinear regimes obtained with the numerical model. They are classified as follows with increasing vortex strength $\Delta Q_{T c}$ (the critical value for jet crossing):

- regime 1 for $\left|\Delta Q_{T}\right|<\Delta Q_{T c}$ (here $\Delta Q_{T c}=1.3$ ), the anticyclone is too weak to cross the jet and it remains north of the jet; after an adjustment period, it is advected eastward. For weak vortices, initially distant from the jet axis, the vortex trajectories and meander amplitudes are well described by the analytical theories (asymptotic expansion) of Stern and Flierl (1987) and of Bell (1990).

- regime 2 for $\Delta Q_{T c}<\left|\Delta Q_{T}\right|<\Delta Q_{T c}+\delta q$ (here $\delta q=0.4<\Delta Q_{T c}$ ), the anticyclone induces meanders on the jet, crosses it, pairs with the cyclonic meander south of the jet and forms a dipole. This process is accompanied by strong filamentation and trapping of ambient fluid as described by Stern (1991). The dipole propagates in directions which depend on the relative strengths of the two vortices, themselves much influenced by filamentation (Figure $2 \mathrm{~b}$ ).

- regime 3 for $\left|\Delta Q_{T}\right|>\Delta Q_{T c}+\delta q$, the anticyclone crosses the jet without dipole formation; indeed the cyclonic meander forming south of the jet is too rapidly elongated to form a strong coherent structure. The anticyclone is then advected eastward, south of the jet axis.

These three regimes exist for a large range of deformation radii but the critical values $\left|\Delta Q_{T c}\right|$ for jet crossing are sensitive to this parameter. We now analyze these regimes.

\section{Absence of jet crossing and steady propagation north of the jet}

Weak vortices $\left(0<\left|\Delta Q_{T}\right|<\Delta Q_{T c}\right)$ are barely eroded during their interaction with the jet (regime 1). After an adjustment period, the vortex propagates eastward north of the jet axis and after a short time becomes phase-locked with a meander (Figure 3a). If the jet meander and the vortex form a stationary state, their drift speed $\left(c_{x}, c_{y}\right)$ can be obtained by minimizing the functional

$$
F\left(c_{x}, c_{y}\right)=\iint\left|J\left(\psi+c_{x} y-c_{y} x, Q\right)\right|^{2} d x d y
$$

Since numerical experiments show eastward propagation of the vortex and meander, $c_{y}$ is expected to be negligible compared with $c_{x}$. In that case, the adjustment towards a quasi stationary state is shown by plotting the scatter plot of its potential vorticity $Q$ versus the relative streamfunction $\psi+c_{x} y$. At an early time $(t=80)$, this relation is dispersive (see Figure $3 \mathrm{~b}$ ). At $t=320$, which corresponds to the final state, minimal dispersion is observed; the drift speeds are then $c_{x}=1.810^{-2}$ and $c_{y} / c_{x}=4.510^{-2}$, which confirms our assumption of a mainly zonal motion. A quasi-stationary state is attained.

This vortex also creates an invariant perturbation of the jet; this perturbation can be 
characterized by its Fourier decomposition (Figure 3c). The largest modal amplitude corresponds to mode $n=2$, followed in intensity by $n=1$ and $n=3,\left(n=k L_{x} / 2 \pi\right)$. These modes are found whatever $\Delta Q_{T}$ in the range given in table 1. Applying this to the Azores Current, with a deformation radius of $30 \mathrm{~km}$, a radius of maximum velocity of the eddy of $40 \mathrm{~km}$, we obtain a wavelength of $375 \mathrm{~km}$ for the jet meander. This value is comparable with that $(400 \mathrm{~km})$ found for the Azores Current crossed by a meddy, using successive hydrological surveys (see Figure 1.8 of Vandermeirsch, 1999).

\section{Jet crossing and subsequent eddy evolutions}

For vortices with intensity close to the critical value $\Delta Q_{T c}$, eastward propagation, north of the jet axis, ceases after a short time: the vortex merges with the jet. A vortex with slightly stronger intensity can cross the jet. Figure 4 a shows the successive steps in that process: (1) the anticyclone is advected eastward by the jet, (2) the anticyclone pushes the jet axis northward upstream and southward downstream, thus creating a cyclonic vortex downstream (and a weaker anticyclone upstream). The cyclone and anticyclone thus form a dipole. (3) Due to the angle of the dipole axis, its average motion is southwestward (therefore across the jet axis).

In regime 2, the anticyclone pairs with the cyclone as a dipole. The size of the cyclone conditions the trajectories of this dipole; this size strongly depends on the crossing velocity and on the filamentation occuring during and after jet crossing (see also beginning of this section). Figure $2 \mathrm{~b}$ presents the three trajectories of regime two obtained with the numerical model as a function of the anticyclonic $\left(\Gamma_{A}\right)$ and cyclonic $\left(\Gamma_{C}\right)$ intensities. When $\Delta Q_{T}$ is close to $\Delta Q_{T c}$, the cyclone is not strongly deformed by the anticyclone and the dipole trajectory is mostly cyclonic until it is split apart by the shear of the jet $\left(\Gamma_{C}>\Gamma_{A}\right)$. Increasing $\Delta Q_{T}$, a value is reached where the filamentation processes affect the two vortices equally; they have equal strengths finally $\left(\Gamma_{C} \approx \Gamma_{A}\right)$ and they translate regularly away from the jet (southward or southwestward). As the anticyclone intensity is again increased, its crossing of the jet is faster so that the southward meander is strongly elongated in the shear flow of the anticyclone. The dipole trajectory is anticyclonic before it is destroyed by the shear of the jet $\left(\Gamma_{C}<\Gamma_{A}\right)$. For all asymmetric dipoles which split apart, the final trajectory of the anticyclone is eastward south of the jet axis, the cyclone moving more slowly in the same direction.

Two examples of (asymmetric and symmetric) dipole formation are shown:

For $\Delta Q_{T}=-1.56$ (Figure $\left.4 \mathrm{~b}\right)$, the dipole slowly rotates cyclonically and resists breaking for a long time. The cyclone has trapped fluid originating from the north of the jet (see again Stern, 1991). The negative vorticity seen around this cyclone results from the wrapping of a filament. The splitting of the dipole (at $t=1100$ ) is due to the increasing shear exerted by the jet, as the dipole moves closer to the jet axis.

For $\Delta Q_{T}=-1.65$ (Figure $4 \mathrm{c}$ ), the crossing of the jet is slightly more rapid than for the previous case, since the anticyclone is stronger ( $\mathrm{cf} t=80)$. Filamentation leads to a cyclone (which splits into two fragments in the shear exerted by its anticyclonic companion) and an anticyclone with similar strengths (as defined hereabove), so that the trajectory remains southwestward. Again the cyclone is surrounded by negative 
vorticity.

In regime 3 , the anticyclone crosses the jet without dipole formation; cyclonic vorticity torn from the jet is completely sheared by the anticyclone, and forms a filament (also see beginning of this subsection).

\subsection{Analytical criterion for jet crossing}

We establish here a simple physical criterion for jet crossing by the vortex. The schematic diagram (Figure 4a) suggests that when the vortex crosses the jet, their maximum velocities must be equal and opposite, so that locally a meridional motion can dominate (occlusion of the southward meander as a cyclone or a filament). The critical condition for jet crossing is thus stated mathematically:

$$
u_{J}(y=0)=-v_{\theta, T}\left(r=R_{T}\right)
$$

which yields a critical vortex intensity $\Delta Q_{T c}$

$$
\frac{\left|\Delta Q_{J}\right|}{\left|\Delta Q_{T c}\right|}=2 \gamma R_{T} I_{1}\left(\gamma R_{T}\right) K_{1}\left(\gamma R_{T}\right)
$$

The asymptotic limit of this quantity, when $\gamma R_{T} \rightarrow \infty$, is $2 \gamma R_{T} I_{1}\left(\gamma R_{T}\right) K_{1}\left(\gamma R_{T}\right) \rightarrow 1$. Note also that the velocity condition forbids jet crossing by cyclones initially located north of the jet axis. Physically, a cyclone would induce an anticyclonic meander on the jet and their pairing would propel the cyclone north-eastward.

The critical condition for jet crossing is plotted on figure $4 \mathrm{~d}$, and the results of numerical experiments, for jet crossing by the anticyclone, are superimposed. The agreement is surpringly good, considering the simplicity of the criterion; indeed, the error $^{3}$ is at most $15 \%$. The interest of this analytical criterion (relation $(6)$ ) is to be dimensionless and to allow a good estimate of $\left|\Delta Q_{J}\right| /\left|\Delta Q_{T c}\right|$ according to $\gamma R_{T}$ or reciprocally.

In fact, this analytical criterion is identical to the existence of a saddle-point of the relative streamfunction $\psi+U y$ on the jet axis, where $U$ is the zonal velocity of the vortex center (see also Bell \& Pratt, 1992). Jet crossing is possible when the saddlepoint in relative streamfunction lies on the jet axis or south of it. The difference with Bell \& Pratt (1992) is that the jet is stable here contrary to their case. Crossing of the jet by the vortex can be strongly affected by jet instability as described in part II. Fast development of unstable meanders can prevent or accelerate this jet crossing.

\subsection{Sensitivity to physical parameters}

We test the sensitivity of this interaction and of the crossing criterion to several physical parameters.

\subsection{1 the initial distance between the vortex and the jet}

On the f-plane, interaction between the jet and the vortex occurs only when they are very close (less than two deformation radii apart). Southward advection of the

\footnotetext{
${ }^{3}$ a $L_{\infty}$ norm of the relative difference between the analytical and numerical results
} 
vortex in this case can only be performed via its interaction with a meander of the jet. The amplitude of this meander (and consequently the intensity of the southward advection) will be weak if the vortex is initially far away from the jet. The influence of dissipation on this interaction is limited in all cases, and the critical vortex intensity for jet crossing varies at most by $10 \%$ with the distance (still $y_{T} \leq 2 R_{d}$ ). For larger initial distances, the vortex does not reach the jet axis, whatever its strength.

\subsection{2 the potential vorticity distribution}

The phenomenology of jet-vortex interaction is not strongly modified if the vortex and jet have continuous potential vorticity distributions, as long as both features remain fairly concentrated and stable. The crossing criterion is still that vortex and jet peak velocities be equal and opposite signed. Barotropically unstable vortices can be strongly altered or break before they reach the jet axis: indeed the sheared jet velocity excites elliptical modes of perturbation on the vortex, which are often the most unstable modes.

\subsection{3 the influence of $\beta$-effect}

For oceanic applications, the influence of $\beta$-effect is now considered. In the absence of the jet, an anticyclone would drift southwestward on the $\beta$-plane with a velocity proportional to $\beta R_{d}^{2}$. This may suggest that anticyclones would always cross eastward jets. In fact, it is not so for three reasons: (1) the criterion for jet crossing is that the zonal velocities of the jet and vortex be equal and opposite; the meridional drift velocity of the vortex is important only to determine the crossing time (2) when $\beta$-effect increases, the vortex radiates energy away as Rossby waves and becomes weaker when it encounters the jet, (3) $\beta$-effect restores zonal streamlines and thus acts against the formation of meanders.

Starting with piecewise-constant jet and vortex, we assume that the vortex still has uniform PVA when it encounters the jet (this is acceptable only if the vortex is not too far away initially); if the vortex has PVA $\Delta Q_{T}$ when it encounters the jet at latitude zero, its original vorticity is therefore $\Delta Q_{T}+\beta y_{T}$. Using this value in the $f$-plane criterion, the comparison with numerical results on the $\beta$-plane is satisfactory (Figure $4 \mathrm{e})$. On this figure, the dimensionless value $\beta=0.046$ is chosen as four times that at mid-latitudes, to obtain a noticeable influence of $\beta$-effect. In conclusion, $\beta$-effect in the ocean is not sufficient to make all vortices cross strong zonal jets.

\section{Eddy interaction with a zonal jet in the shallow- water model}

The study of section 3 is now generalized in the shallow-water model, where frontal and nonlinear jets and vortices can be adequately represented. The initial conditions of the numerical model are identical to those of section 3.1. Here we concentrate on frontal effects (i.e. $R o / B u \sim 1$ ), while keeping the Rossby number moderate $($ Ro $<0.25)$. Thus, large isopycnal deviations across the jet and cyclone/anticyclone asymmetry are allowed. First, the results of numerical experiments of anticyclone- 
jet interaction are described and compared with the quasi-geostrophic results; then, cyclone-anticyclone asymmetry is investigated.

\subsection{Nonlinear regimes of jet-vortex interaction}

Firstly, we have checked that both the zonal jet and the circular vortex are also stable in the shallow-water model; indeed, a small disturbance added to these mean flows does not grow in a nonlinear simulation with minimal dissipation, at least for the range of $R o$ and $B u$ considered in this study (see table 2).

The three regimes are similar to those obtained in the QG model (cf Figures 2a and $2 b)$. Numerical simulations of jet-vortex interactions show that nonlinear regimes in the SW model bear strong similarity with those in the QG model:

- regime 1 for $\left|\Delta q_{T}\right|<\Delta q_{T c}$ (here $\Delta q_{T c}=1.3$ ): weak vortices do not cross the jet, and drift steadily along it. Later, the vortex is slightly deformed elliptically and the jet undergoes a long-wave perturbation. The vortex and jet perturbation move at the same speed. The vortex trajectory has become parallel to the jet axis. The PVA has identical isolines as the Bernoulli function in the moving frame of reference: $B=p \rho_{0}+12\left[\left(u-c_{x}\right)^{2}+v^{2}\right]+f_{0} c_{x} y$. The final state of jet and vortex is thus stationary (not shown).

- regime 2 for $\Delta q_{T c}<\left|\Delta q_{T}\right|<\Delta q_{T c}+\delta q$ (here $\delta q=0.4$ ): the three trajectories of regime two, found in case $\mathrm{QG}$, are also present in the $\mathrm{SW}$ model (Figure 2b). The subsequent trajectories of this dipole depend on the intensity of the initial vortex.

- regime 3 for $\left|\Delta q_{T}\right|>\Delta q_{T c}+\delta q$ : the anticyclone crosses the jet and simply drifts eastward along it without pairing with the cyclone. This behavior is very similar to that observed in the QG model.

As in the quasi-geostrophic model, varying the initial distance between the vortex (again within a limited range) and jet or adding $\beta$-effect does not qualitatively modify the jet-vortex interaction.

\subsection{Analytical criterion for jet crossing}

We generalize the crossing criterion from the QG to the SW model, without the cyclostrophic term for the vortex (this will be discussed later on). As in the QG model the condition for jet crossing is stated mathematically like (5), which yields:

$$
\frac{\left|\Delta q_{J}\right|}{\left|\Delta q_{T c i}\right|}=\frac{H_{i}}{H} 2 \gamma_{m} R_{T} I_{1}\left(\gamma_{T i} R_{T}\right) K_{1}\left(\gamma_{T i} R_{T}\right)
$$

where $i=1$ stands for the anticyclonic case, $i=2$ for the cyclonic case, $\gamma_{m}$ is a mean inverse radius of deformation (see Appendix), and $H_{1}$ and $H_{2}$ are the thicknesses to the north and south of the jet (see appendix for mathematical details).

With $\gamma R_{T}$ large, the asymptotic limit of relation (7) is, in first approximation, $H_{1} / H=0.75$ and $H_{2} / H=1.5$ with $\Delta q_{J}=1$. Relation (7) is defined only for $\Delta q_{T c i}>-f_{0} / H_{i}$ : this constraint prevents inertial or hydrostatic instabilities. This also imposes a limit on the intensity of the anticyclone: $\Delta q_{T c 1}>-2$. Relation (7) is implicit in $\Delta q_{J}$ and $\Delta q_{T c i}$ since $\gamma_{m}$ and $\gamma_{T i}$ are functions of $\Delta q_{J}$ and $\Delta q_{T c i}$.

On figures $5 \mathrm{a}$ and $5 \mathrm{~b}$, we compare the analytical criterion for jet crossing (without cyclostrophic term) to the exact one (with cyclostrophic term), which can be obtained 
only numerically. The comparison is very satisfactory, the errors are at most $2 \%$ for anticyclones and $10 \%$ for the cyclones. These results confirm our assumption that the cyclogeostrophic term is weak if $R o \leq 0.25$. The limits of $\left|\Delta q_{J}\right| /\left|\Delta q_{T c i}\right|$ on figures 5a and $5 \mathrm{~b}$ for $\gamma R_{T}$ large are about 0.9 for the anticyclone and 1.4 for the cyclone ; these limits are in good agreement with the asymptotic limit of equation (7), 0.75 and 1.5 .

We now compare, on figure $5 c$, the analytical criterion and the results of nonlinear numerical experiments of jet-vortex interaction in the SW model: the error is at most $14 \%$ for anticyclones and cyclones : cyclones cross the jet more easily than anticyclones. This asymmetry is detailed in the following subsection.

\subsection{Cyclone-anticyclone asymmetry}

To physically understand the asymmetry between cyclones and anticyclones, several physical reasons can be called upon:

- the ambient radius of deformation north of the jet $R_{d 1}=1 / \gamma_{1}$ is smaller than south of it $R_{d 2}=1 / \gamma_{2}$ because of the large deviations of density interface associated with the jet. Therefore, for the same vortex (either a cyclone or an anticyclone), the velocity field will be more concentrated north of the jet than south of it. In the Gulf-Stream area, the deformation radius is $15 \mathrm{~km}$ north of the jet and $35 \mathrm{~km}$ south of it (Blayo, 1992).

- for strong vortices, the local deformation radius is due both to the ambient stratification and to the interface deviation associated with the vortex.

- cyclostrophy could be another source of asymmetry between cyclones and anticyclones, but we have seen that it does not play a major role for the crossing criterion, as long as $R o$ is moderate.

To illustrate the cyclone-anticyclone asymmetry, we present two experiments located in regime 3: we set $\left|\Delta q_{J} / \Delta q_{T i}\right|=0.6$. The cyclone crosses the jet more rapidly than the anticyclone (Figures $5 \mathrm{~d}$ and $5 \mathrm{e}$ ); this is in agreement with the criterion for jet crossing: the critical intensity of the cyclone needs to be smaller than that of the anticyclone.

Furthermore, the anticyclonic fragment torn away by the cyclone from the jet has a size comparable with $R_{d 2}$, larger than the cyclonic one entrained by an anticyclone, which has $R \sim R_{d 1}$. Though the initial trajectories of the cyclone (Figure $5 e$ ) and of the anticyclone (Figure 5d) are comparable, both advected eastward by the opposite-sign meander, their final trajectories are quite different: the anticyclone moves southward, with a cyclonic companion of equal strength, while the cyclone rotates around the strong anticyclonic patch that it has torn away from the jet (see the similarity with the quasi-geostrophic result on figure $4 \mathrm{~b}$ ).

We now look for such an asymmetry in the initial conditions of the experiments. We plot the meridional sections of the thickness $H$, velocity $U$ and PVA $\Delta q$ for the jet and vortex in the former two experiments (see figures $5 \mathrm{f}$ and $5 \mathrm{~g}$ ). In the absence of the jet, the velocities of the anticyclone are 2.6 times larger than those of the cyclone. At first glance, this stronger intensity of anticyclones contradicts the predominance of cyclones in jet crossing. But in the presence of the jet, the difference in intensity is not only compensated but slightly reversed: the maximum velocity of the cyclone is now $10 \%$ larger than that of the anticyclone. Furthermore, the 
asymmetry in deformation radii: $R_{d 2} / R_{d 1}=\sqrt{H_{2} / H_{1}}=\sqrt{2}$ leads to an asymmetry in the sizes of torn fragments. Fluid originating from south of the jet will have a larger radius of deformation (and thus should acquire a larger size) than that coming from north of the jet. Therefore, this north-south asymmetry in stratification plays a dominant role in the parity bias between cyclone and anticyclone interaction with the jet. This dominant role is obviously related to our choice of a frontal jet $(R o / B u \sim 1)$.

Finally, we consider again cyclone and anticyclone interaction with the jet, but now in regime 1 , by setting $R_{T}=1.2$ and $\left|\Delta q_{J} / \Delta q_{T i}\right|=1$. In both experiments, the vortices do not cross the jet and are advected along the current (Figures $5 \mathrm{~h}$ and $5 \mathrm{i}$ ). A difference is noticeable between the two experiments: the deformation of the jet by the cyclonic vortex is of greater amplitude. Again, this is due to the stronger velocity field of the cyclone.

\section{Conclusions}

The interaction between a vortex and a zonal jet has been studied in two one-and-ahalf layer models, respectively with quasi-geostrophic and with shallow-water equations. The jet and the vortex have piecewise-constant potential vorticity. Numerical simulations with both models show that weak vortices drift quasi-steadily along the jet, while strong vortices can cross the jet and tear an opposite-sign vorticity patch (often a vortex, sometimes a filament). These evolutions are generic: experiments performed with jets and vortices having continuous potential vorticity profiles show comparable results, as long as barotropic instability is excluded.

In most cases on the $f$-plane (with or without jet crossing), the final vortex trajectory is eastward and stationary relative to the jet, except when the initial vortex and its opposite-sign companion have identical strengths. Stationarity of teh final state is evidenced by plotting the relative streamfunction (or the Bernoulli function) versus potential vorticity. When parameters are chosen to represent the Azores Current, the meanders created by the vortex on the jet have a characteristic wavelength close to $400 \mathrm{~km}$ (as observed for the Azores Current).

The crossing of the jet by the vortex can be predicted analytically: the crossing criterion is derived from the physical observation that the vortex must have at least equal and opposite velocity to that of the jet on its axis. This condition on velocity leads to a relation between jet and vortex intensities, vortex and deformation radii. For large of $\gamma R_{T}$ the limit of this criterion states that the jet and vortex must have identical potential vorticities for crossing to occur. The equal and opposite velocity condition for crossing also applies in the shallow-water model. The analytical criterion in the SWE model exhibits cyclone-anticyclone asymmetry: cyclones can cross the jet more easily than anticyclones.

Kielmann et al. (1987) analyzed the thermal front associated with the Azores current, from infra-red images. He observed a meridional asymmetry marked by strong anticyclonic meanders and by a warm water anomaly north of the jet. This mesoscale 
activity was confirmed by Traon et al. (1994) with altimetric data. In our model, it was shown that the north-south asymmetry results mostly from different local deformation radii. But this asymmetry remains moderate and does not alter the qualitative behavior observed in the quasi-geostrophic model.

This process study does not claim to be comprehensive, but to describe and characterize the physical evolutions of a simple jet and vortex flow. An interesting extension of this study involves unstable jets and baroclinic effects. For instance, baroclinic instability of the jet can occur when the jet has opposite sign potential vorticity in two active layers. This is the subject of the second part of this paper, with an application to meddies crossing the Azores Current.

\section{Acknowledgements}

Funding for this study was provided by SHOM and DGA under programs FRE 91009401 and PEA 982401. The authors express gratitude to Dr Bach Lien Hua for fruitful discussions. The present results are part of FOV's doctoral dissertation at the Université de Bretagne Occidentale (1999). This work is a contribution to the SEMAPHORE research program.

\section{Appendix : Analytical velocity fields in the SW model}

We define the potential vorticity anomaly $\delta q_{J}$ of the jet by:

$$
\delta q_{J}=\frac{\zeta_{J}-f_{0} \eta_{J} / H}{H+\eta_{J}}
$$

i.e. the difference between total potential vorticity and that of a state of rest, in the absence of the jet, $f_{0} / H$. We first calculate the variation of layer thickness $\eta_{J}$ associated with a jump in potential vorticity anomaly $\Delta q_{J}$ defining the zonal jet in the absence of the vortex:

$$
\delta q_{J}(y)=\left\{\begin{aligned}
\Delta q_{J} / 2 & , y>0 \\
-\Delta q_{J} / 2 & , y<0
\end{aligned}\right.
$$

The geostrophic balance leads to:

$$
\left(\nabla^{2}-\gamma_{J}^{2}\right) \eta_{J}=a H \delta q_{J}
$$

where $\gamma_{J}^{2}=\gamma^{2}\left(1+H \delta q_{J} / f_{0}\right)$ and $a=f_{0} / g^{\prime}$.

The variation of layer thickness $\eta_{J}$ is given by:

$$
\eta_{J}(y)=a H \Delta q_{J} \begin{cases}\left(2 \gamma_{1} \gamma_{m}\right)^{-1} \exp \left(-\gamma_{1} y\right)-\gamma_{1}^{-2} & , y>0 \\ -\left(2 \gamma_{2} \gamma_{m}\right)^{-1} \exp \left(\gamma_{2} y\right)+\gamma_{2}^{-2} & , y<0\end{cases}
$$

We now obtain the velocity $u_{J}(y)=-a^{-1} d \eta_{J} / d y$ :

$$
u_{J}(y)=H \Delta q_{J} /\left(2 \gamma_{m}\right) \exp \left(-\gamma_{J}|y|\right)
$$


with $\gamma_{m}^{-} 1=2\left(\gamma_{1} / \gamma_{2}+\gamma_{2} / \gamma_{1}\right) /\left(\gamma_{1}+\gamma_{2}\right)$ and $\gamma_{i}^{2}=f_{0}^{2} /\left(g^{\prime} H_{i}\right)=\gamma^{2} H / H_{i} . H_{1}$ and $H_{2}$ are the thickness the north and south of the jet. $H_{1}$ and $H_{2}$ are defined mathematically with the limit of $H+\eta_{J}(y \rightarrow \pm \infty)$ :

$$
H_{i} / H=1-\left[f_{0} /\left(H \delta q_{J}\right)+1\right]^{-1}
$$

We define the potential vorticity anomaly $\delta q_{T}$ of the vortex by:

$$
\delta q_{T}=\frac{\zeta_{T}-f_{0} \eta_{T} / H_{i}}{H_{i}+\eta_{T}}
$$

i.e. the difference of total PV and of ambient PV. For the anticyclone (resp. for the cyclone), located north (resp. south) of the jet, the surrounding $\mathrm{PV}$ is $f_{0} / H_{1}$ (resp. $\left.f_{0} / H_{2}\right)$.

We now consider the vortex, defined by a circular patch of constant potential vorticity anomaly $\Delta q_{T}$, in the presence of the jet :

$$
\delta q_{T}(r)=\left\{\begin{aligned}
\Delta q_{T i}, & |r|<R_{T} \\
0, & |r|>R_{T}
\end{aligned}\right.
$$

Neglecting cyclostrophic terms, the variation of layer thickness $\eta_{T}$ obeys the geostrophic balance:

$$
\left(\nabla^{2}-\gamma_{T i}^{2}\right) \eta_{T}=a^{-1} H_{i} \delta q_{T}
$$

where $\gamma_{T i}^{2}=\gamma_{i}^{2}\left(1+H_{i} \delta q_{T} / f_{0}\right)$.

The solution $\eta_{T}$ is given by:

$$
\eta_{T}(r)=a H_{i} \Delta q_{T i} / \gamma_{T i}\left\{\begin{array}{rr}
R_{T} K_{1}\left(\gamma_{T i} R_{T}\right) I_{0}\left(\gamma_{T i} r\right)-\gamma_{T i}{ }^{-1} & ,|r|<R_{T} \\
-R_{T} I_{1}\left(\gamma_{T i} R_{T}\right) K_{0}\left(\gamma_{T i} r\right) & ,|r|>R_{T}
\end{array}\right.
$$

We derive the velocity via $v_{\theta, T}(r)=a^{-1} d \eta_{T} / d r$ :

$$
v_{\theta, T}(r)=\left\{\begin{array}{lll}
H_{i} \Delta q_{T i} R_{T} & K_{1}\left(\gamma_{T i} R_{T}\right) I_{1}\left(\gamma_{T i} r\right), & |r|<R_{T} \\
H_{i} \Delta q_{T i} R_{T} & I_{1}\left(\gamma_{T i} R_{T}\right) K_{1}\left(\gamma_{T i} r\right), & |r|>R_{T}
\end{array}\right.
$$




\section{References}

Bell G.I., 1990 : Interaction between vortices and waves in a simple model of geophysical flow. Phys. Fluids, A, 2 (4), 575-586.

Bell G.I. and Pratt L.J., 1992 : The interaction of an eddy with an unstable jet. J. Phys.Oceanogr., 22, 1229-1244.

Blayo, E., 1992 : Développement d'un modèle quasi-géostrophique de la circulation océanique, Application à l'océan Atlantique Nord. Thèse de Doctorat de l'Université Joseph-Fourier-Grenoble I, Grenoble, France, 191 pp.

Bleck R. and Boudra D.B, 1986 : Wind-driven spin-up in eddy-resolving ocean models formulated in isopycnic and isobaric coordinates. J. Geophys. Res., 91, 7611-7621. Dritschel, D.G. and Saravanan R., 1994 : Three-dimensional quasi-geostrophic contour dynamics with an application to stratospheric vortex dynamics. Q. J. R. Met. Soc., 120, 1267-1297.

Holland W.R., 1978 : The role of mesoscale eddies in the general circulation of the oceans. Numerical experiments using a wind-driven quasi-geostrophic model. J. Phys. Oceanogr., 8, 363-392.

Käse R.H. and Zenk W., 1996 : Structure of the Mediterranean water and meddy characteristics in the Northeast Atlantic. In The Warmwatersphere of the North Atlantic Ocean, ch.12, W. Krauss, Ed., Gebruder Borntraeger, Berlin, 445 pp.

Kielmann J. and Käse R.H., 1987 : Numerical modeling of meander and eddy formation in the Azores current frontal zone. J. Phys. Oceanogr., 17, 529-541.

Le Traon P.Y. and De Mey P., 1987 : The eddy field associated with the Azores front east of the Mid-Atlantic ridge as observed by geosat altimeter. J. Geophys. Res., 99 (C5), 9907-9923.

Orlanski I., 1976 : A simple boundary condition for unbounded hyperbolic flows. J. Comp. Phys., 21, 251-269.

Pratt L.J., 1988 : Meandering and eddy detachment according to a simple (looking) path equation. J. Phys. Oceanogr., 18, 1627-1640.

Pratt L.J. and Stern M.E., 1986 : Dynamics of potential vorticity fronts and eddy detachment. J. Phys. Oceanogr., 16, 1101-1120.

Richardson P.L., 1980 : Gulf Stream ring tracjetories. J. Phys.Oceanogr., 10, 90-104. Richardson P.L. and Tychensky A., 1998 : Meddy trajectories in the Canary Basin measured during the SEMAPHORE experiment, 1993-1995. J. Geophys. Res., 103, C11, 25029-25045.

Stern, M.E., 1985 : Lateral wave breaking and shingle formation in shear flow. J. Phys. Oceanogr., 15, 1274-1283.

Stern M.E., 1991 : Entrainment of an eddy at the edge of a jet. J. Fluid Mech., 228, 343-360.

Stern M.E. and Pratt L.J., 1985 : Dynamics of vorticity fronts. J. Fluid Mech., 161, 513-532.

Stern M.E. and Flierl G.R., 1987 : On the interaction of a vortex with a shear flow. J.Geophys. Res., 92, C10, 10733-10744.

Tychensky A. and Carton X., 1998 : Hydrological and dynamical characterization of meddies in the Azores region: a paradigm for baroclinic vortex dynamics. J. Geophys. Res. C, 103, 25,061-25,079.

Valcke S., 1995 : Coalescence de tourbillons océaniques en milieu stratifié tournant: 
une étude numérique. Thèse, Université Joseph-Fourier, Grenoble, France, 169 pp. Valcke, S. and Verron J., 1996 : Cyclone-anticyclone asymmetry in the merging process. Dyn. Atmos. Oceans, 24, 227-236.

Vandermeirsch, F., 1999 : Interaction entre un jet et un tourbillon oceaniques; application au courant des Acores et aux meddies de la campagne Semaphore. Thèse de Doctorat No 666 de l'Université de Bretagne Occidentale, Brest, France, 207 pp. 


\section{Figure Captions}

Fig. 1:

Schematic initial conditions of jet-vortex interaction.

Fig. 2:

(a) Schematic diagram showing the three dynamic regimes of trajectories for the anticyclone initially located north of the jet as a function of the vortex intensity $\Delta Q_{T}$. The jet axis (PV front) is materialized by dashed lines.

(b) Schematic diagram showing the three trajectories of regime two (Figure 2a) for the anticyclone initially located north of the jet as a function of the anticyclonic $\Gamma_{A}$ and cyclonic $\Gamma_{C}$ intensities.

Fig. 3: (i) Time-series of potential vorticity maps in the horizontal plane for regime 1 $\left(\Delta Q_{T}=-0.6\right)$. The little square denotes the initial position of the vortex and the thin solid line its trajectory. The jet and vortex undergo an adjustment stage until $t=160$, followed by a quasi-stationary evolution;

(ii) Scatter-plots $\left(q, \psi+c_{x} y\right)$ of the vortex for regime 1 during the transient state $(t=80)$ and during the quasi stationary evolution $(t=320)$;

(c) Time-evolution of the first five zonal modes of perturbation on the jet, during the interaction with the vortex $\left(\Delta Q_{T}=-0.6\right)$. Mode $n=2$ is the most representative of the interaction.

Fig. 4:

(a) Schematic diagram showing four stages of jet crossing by a vortex in the quasigeostrophic model and in the shallow-water model;

(b) Potential vorticity maps in the horizontal plane for regime $2 \mathrm{a}\left(\Delta Q_{T}=-1.56\right)$ at times $t=40,80,680$ and 1220 ;

(c) Potential vorticity map in the horizontal plane for regime $2 \mathrm{~b}\left(\Delta Q_{T}=-1.65\right)$ at times $t=160$ and 300. A symmetric dipole is formed and drifts southwestward;

(d) Comparison of the analytical criterion for jet crossing (solid line) with the nonlinear evolutions of the anticyclonic vortex in the numerical quasi-geostrophic model; "N" indicates trajectories north of the jet, "S" represents jet crossing;

(e) Comparison of critical values of vortex intensity for jet crossing with analytical criterion for $\beta=0.046$ (dashed lines with circles), and analytical criterion for $\beta=0$ (solid line).

Fig. 5:

(a) Comparison between the analytical criterion for jet crossing by the anticyclonic vortex (solid line) and the numerical criterion resulting from SWE model (solid line with circles) with respect to stratification. The quasi-geostrophic analytical criterion is recalled (dashed line);

(b) as figure 5a with cyclonic vortex.

(c) Comparison between the analytical criterion for jet crossing (the anticyclones, solid line with stars and the cyclones, solid line with crosses) and the nonlinear evolutions SWE model (the anticyclones, solid line and the cyclones, solid line with dots) with respect to stratification. The quasi-geostrophic analytical criterion is recalled (dashed line);

( $d$ and e) Time series of potential vorticity anomaly in the SWE model for the interaction of an anticyclone (d) or of a cyclone (e) $\left(\left|\Delta q_{J} / \Delta q_{T i}\right|=0.6\right)$ with the zonal jet; ( $f$ and $g$ ) Meridional sections of interface deviation (compared with unity layer thick- 
ness at rest), zonal velocity and potential vorticity anomaly, for the initialisation of the anticyclone without jet (f1, 2 and 3 ) or cyclone without jet (g1, 2 and 3 )and for the initialisation of the anticyclone-jet ( $\mathrm{f} 4,5$ and 6 ) or cyclone-jet ( $\mathrm{g} 4,5$ and 6 ) interaction;

(h and i) Time series of potential vorticity anomaly in the SWE model for the interaction of an anticyclone (h) or of a cyclone (i) $\left(\left|\Delta q_{J} / \Delta q_{T i}\right|=1\right)$ with the zonal jet. 
Tables

\begin{tabular}{|c|c|c|c|}
\hline$\Delta Q_{T}$ & $\gamma$ & $y_{T}$ & $\beta$ \\
\hline \hline$[-6.0,0]$ & {$[0.16,3.0]$} & {$[1.5,2.5]$} & {$[0,0.064]$} \\
\hline
\end{tabular}

Table 1: Ranges of variations for the flow parameters in the quasi-geostrophic model: potential vorticity of the vortex, internal radius of deformation, initial jet-vortex separation and planetary potential vorticity anomaly. Note that $\Delta Q_{J}=1, R_{T}=1$.

\begin{tabular}{|c|c|c|c|}
\hline$\Delta q_{T}$ & $R_{o}$ & $B u$ & $y_{T}$ \\
\hline \hline$[-2.75,2]$ & {$[0.17,0.25]$} & {$[0.06,0.44]$} & {$[1.5,2.5]$} \\
\hline
\end{tabular}

Table 2: Ranges of variations for the flow parameters in the shallow-water model: potential vorticity anomaly of the vortex, Rossby number, Burger number, initial jet-vortex separation. Note that $\Delta q_{J}=1, R_{T}=1$. 


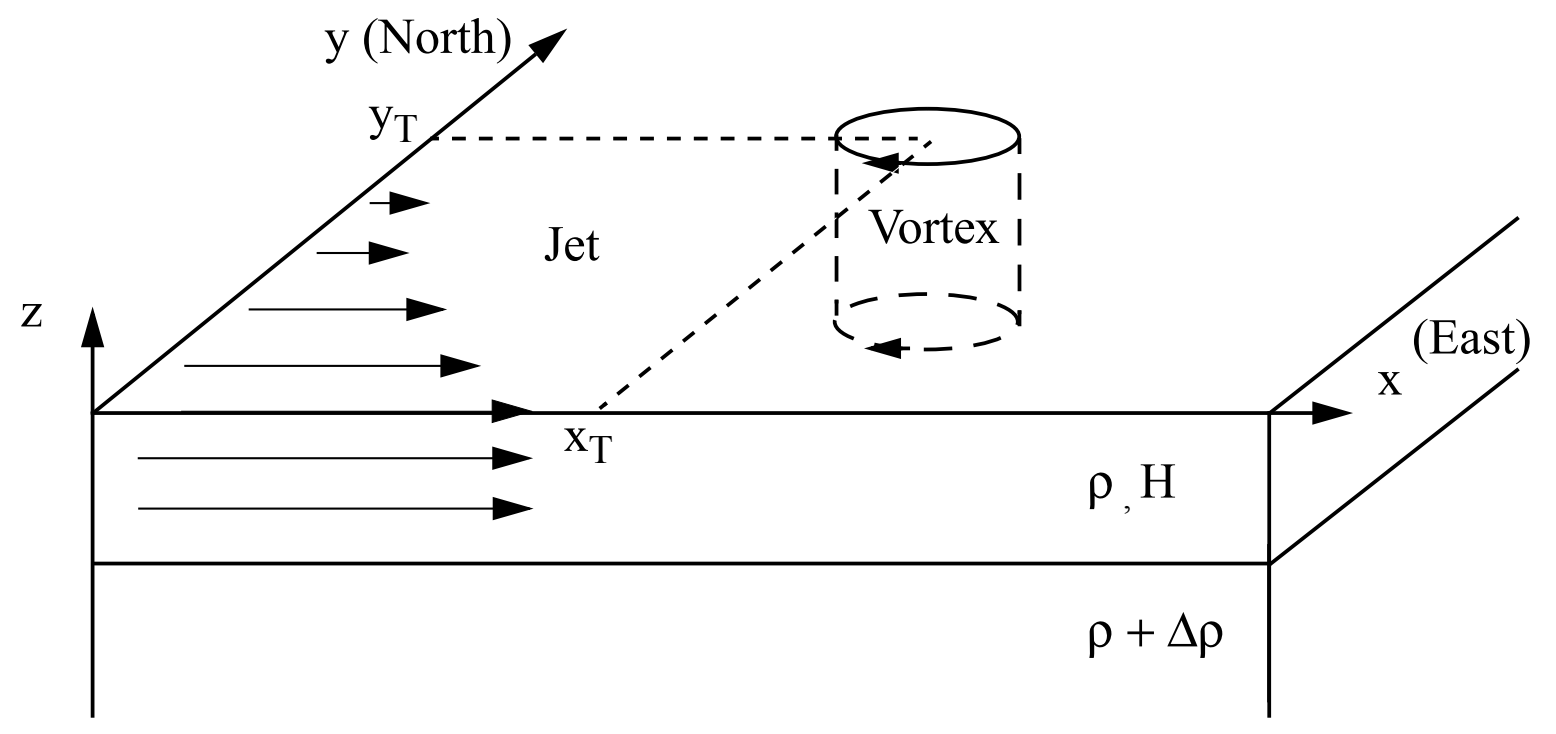

FIG.1 


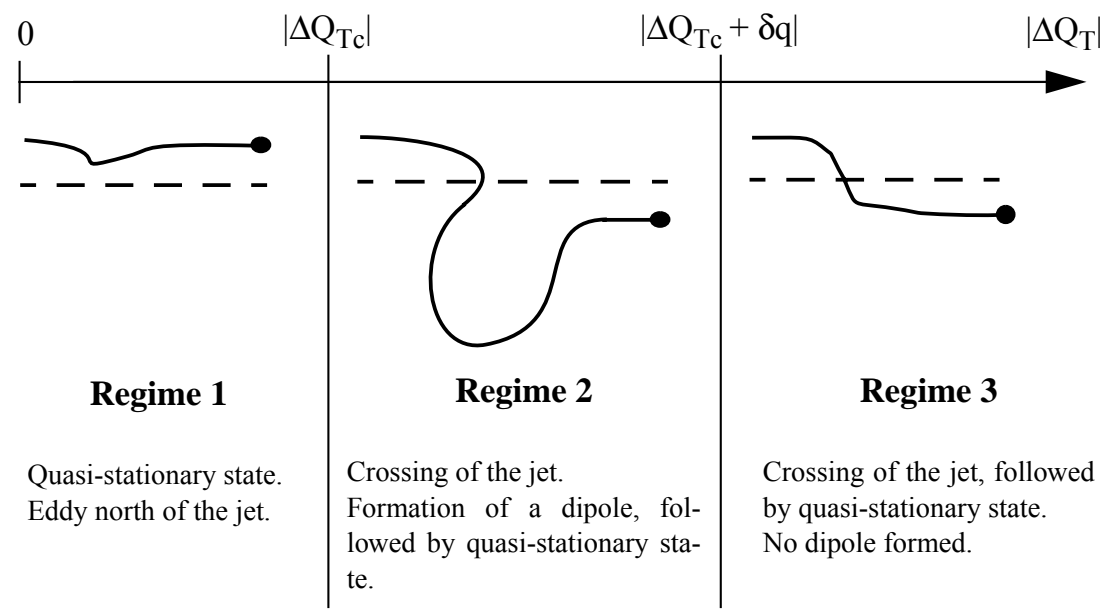

FIG.2a 


\begin{tabular}{l|l|l}
$\Gamma \mathrm{C}>\Gamma_{\mathrm{A}}$ & $\Gamma \sim \Gamma_{\mathrm{A}}$ \\
\hline $\begin{array}{l}\text { Crossing of the jet. } \\
\text { Formation of a dipole, } \\
\text { followed by quasi-statio- } \\
\text { nary state. }\end{array}$ & $\begin{array}{l}\text { Formation of a dipole moving } \\
\text { towards the south-west. }\end{array}$ & $\begin{array}{l}\text { Crossing of the jet. } \\
\text { Formation of a the dipole } \\
\text { moving towards the west, } \\
\text { then quasi-stationary state. }\end{array}$ \\
\hline
\end{tabular}

FIG.2b 

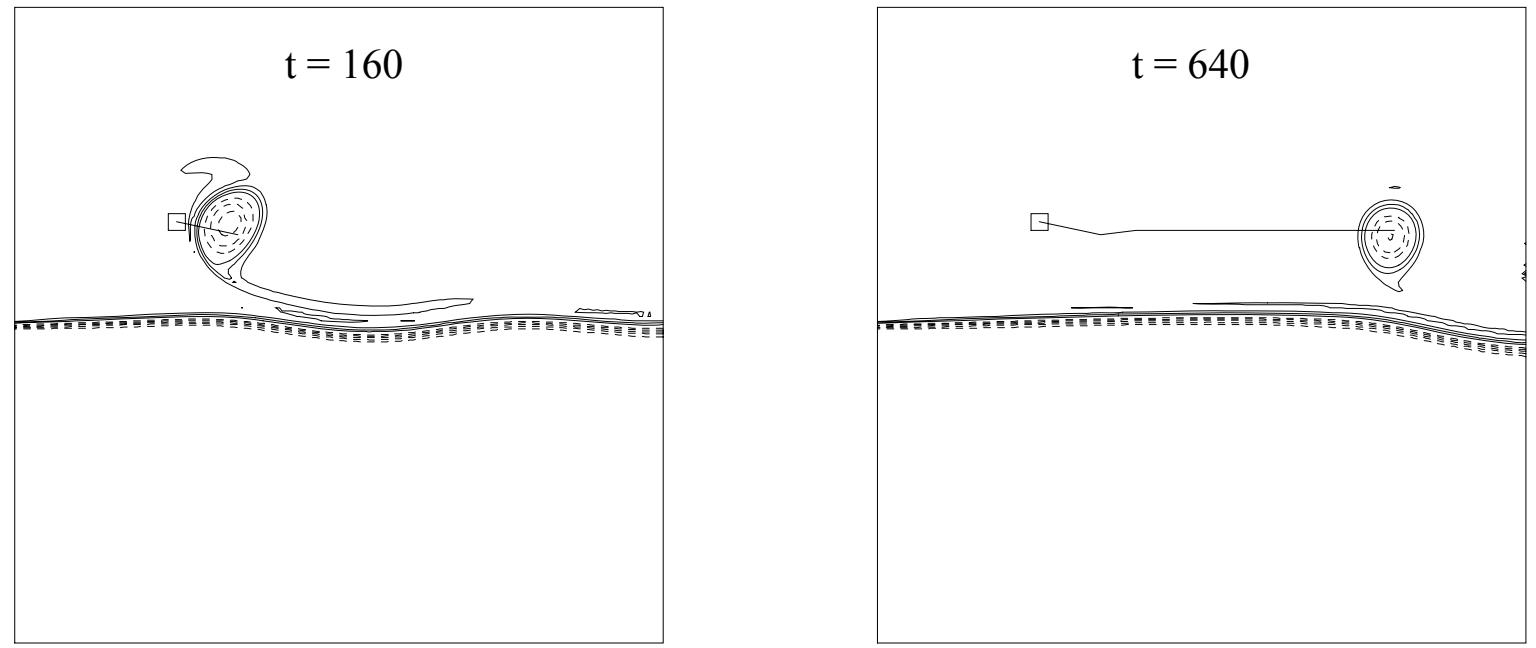

FIG.3a 

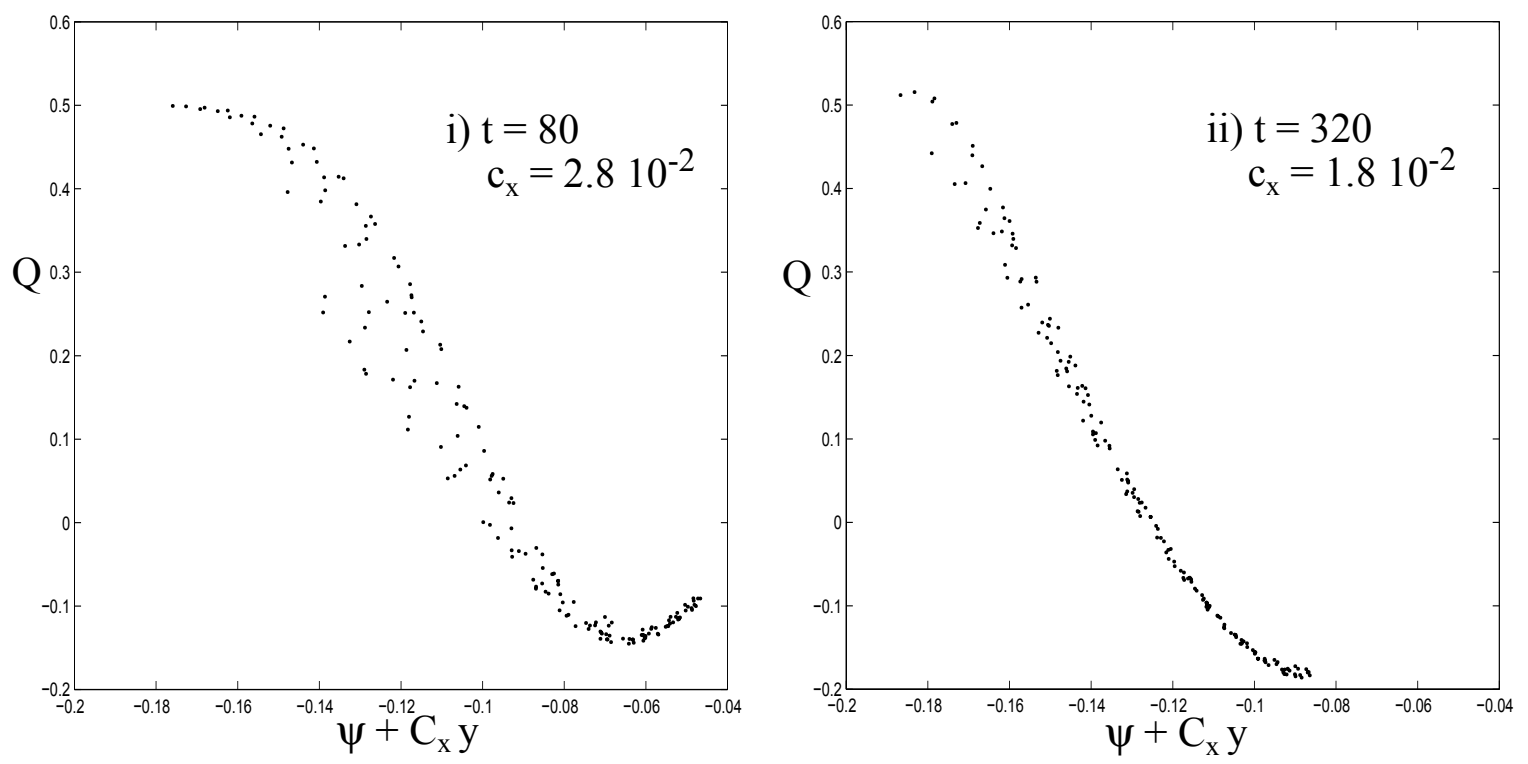

FIG.3b 


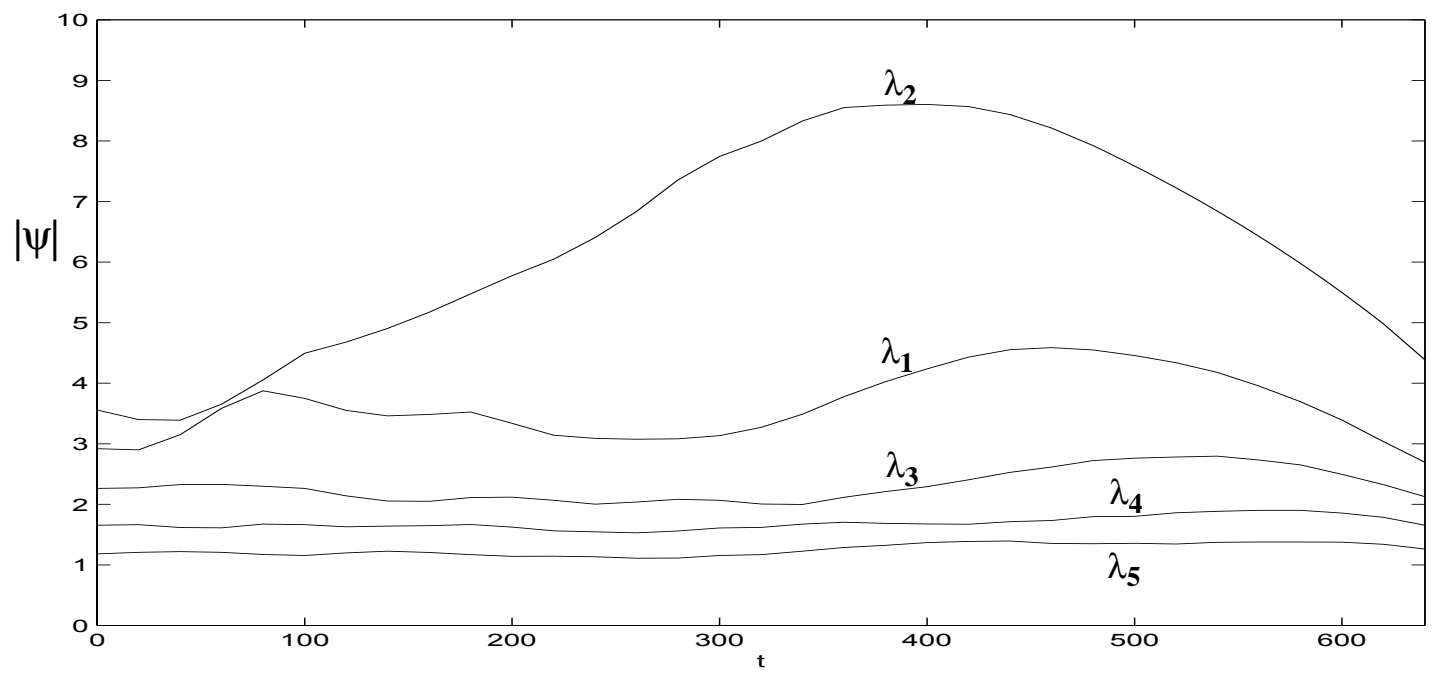

FIG.3c 
1) $t=0$

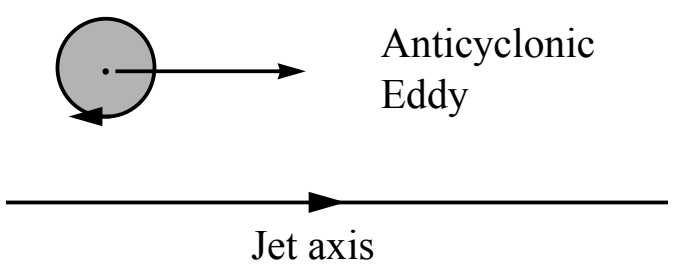

3) $t_{2}>t_{1}$

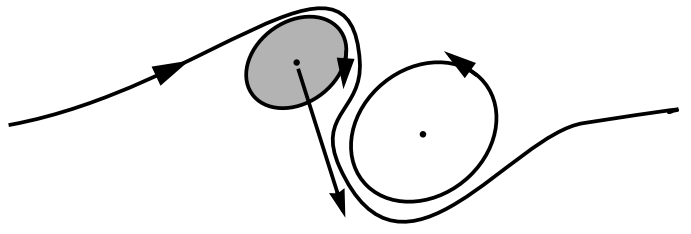

: Advection of the eddy
2) $t_{1}>0$

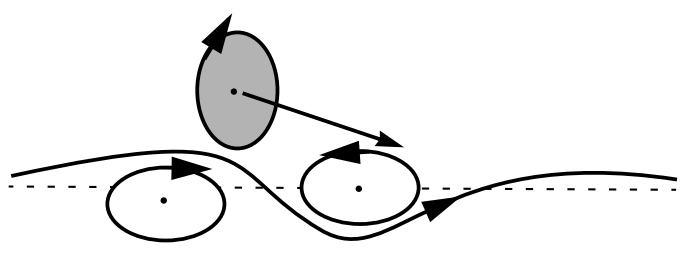

4) $t_{3}>t_{2}$

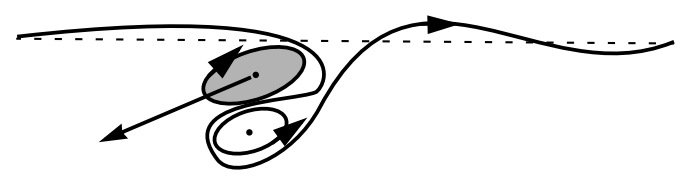

FIG.4a 

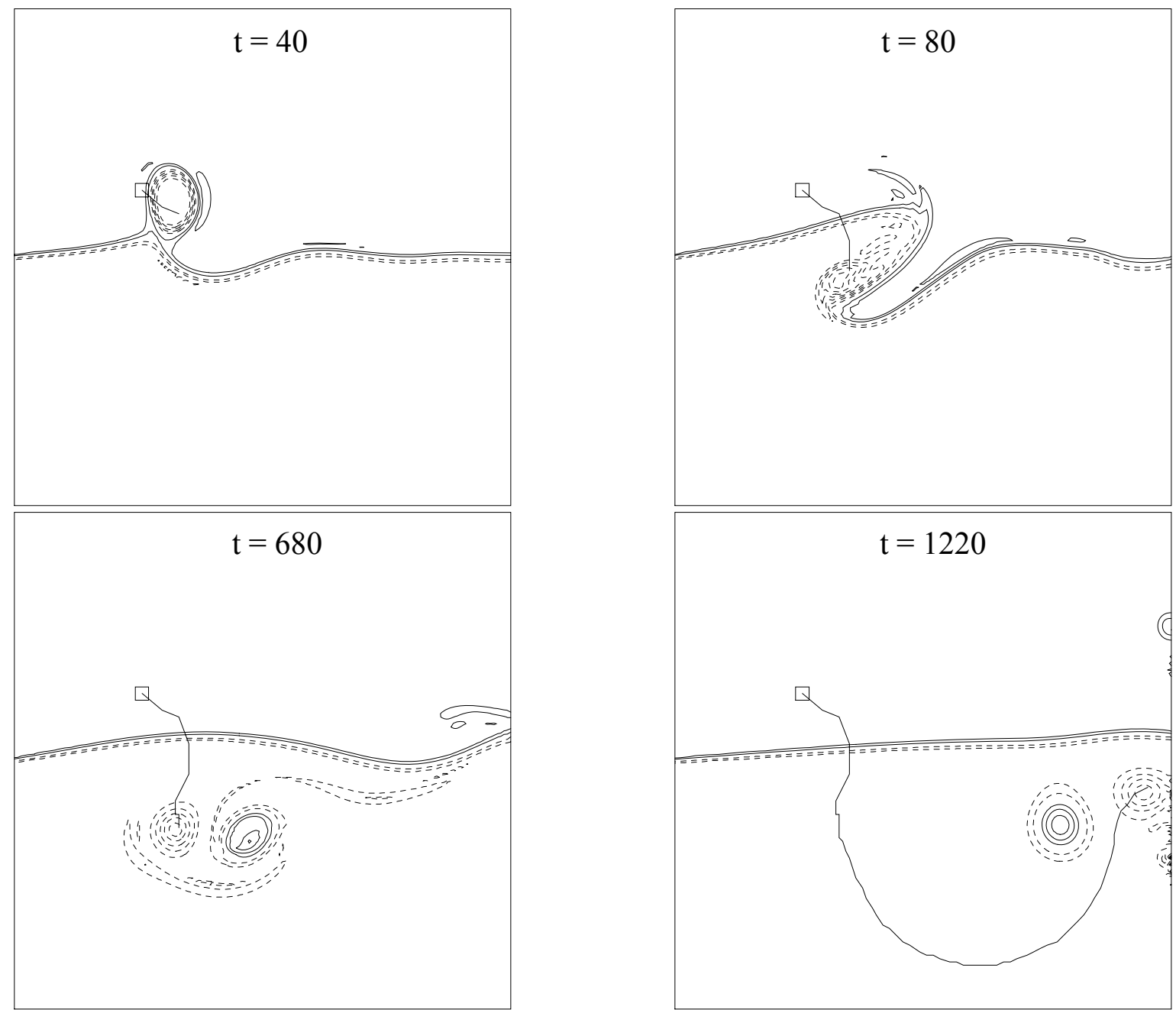

FIG.4b 

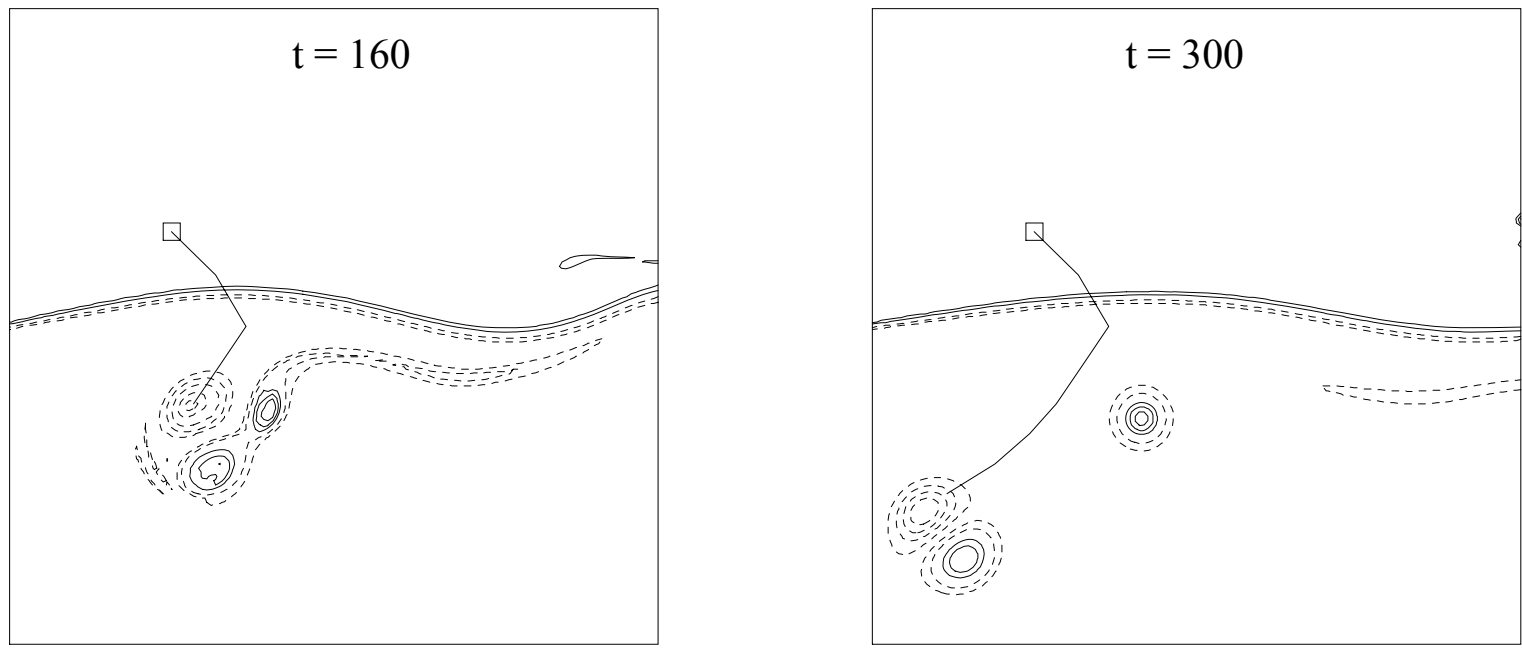

FIG.4c 


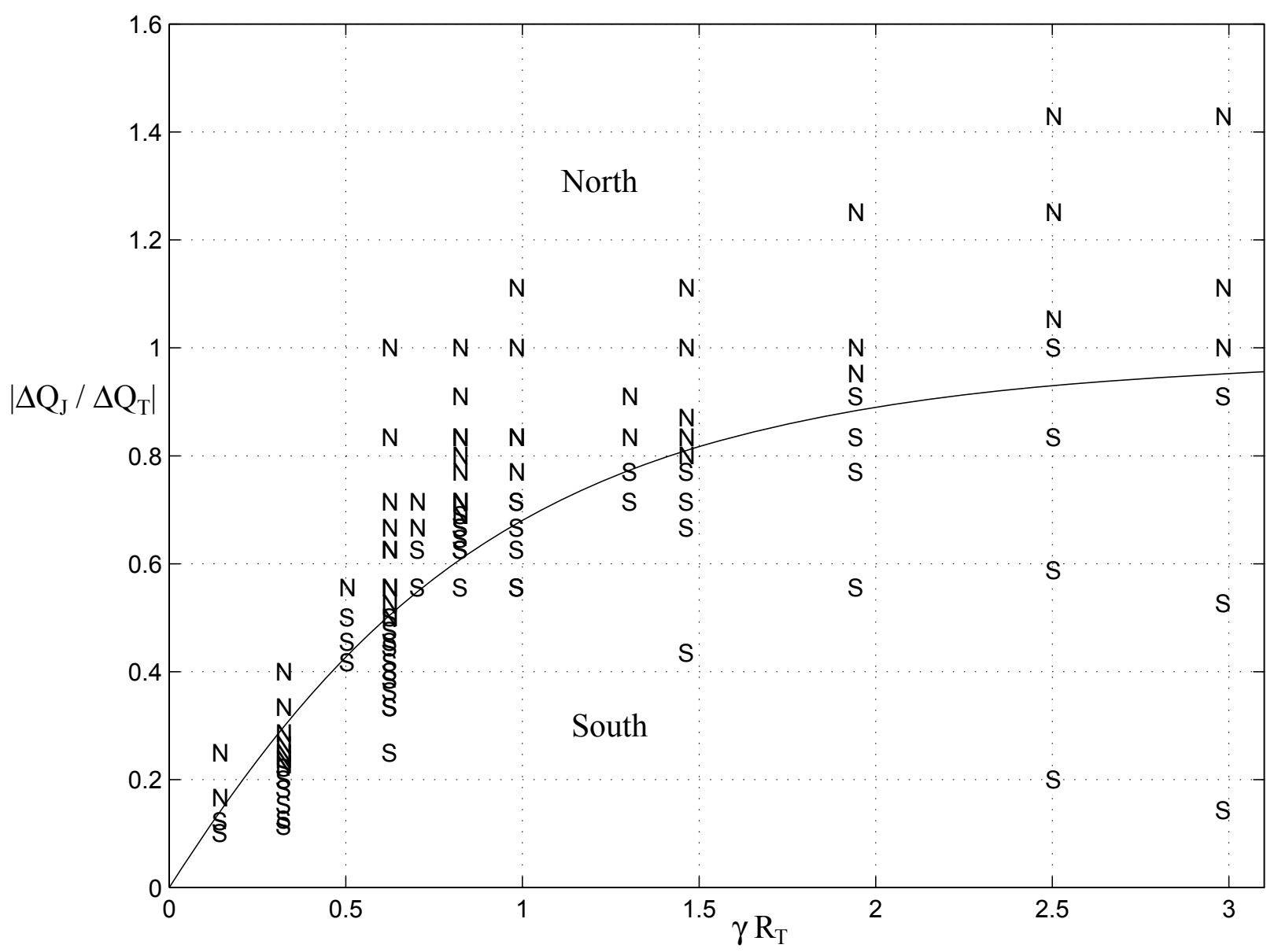

FIG.4d 


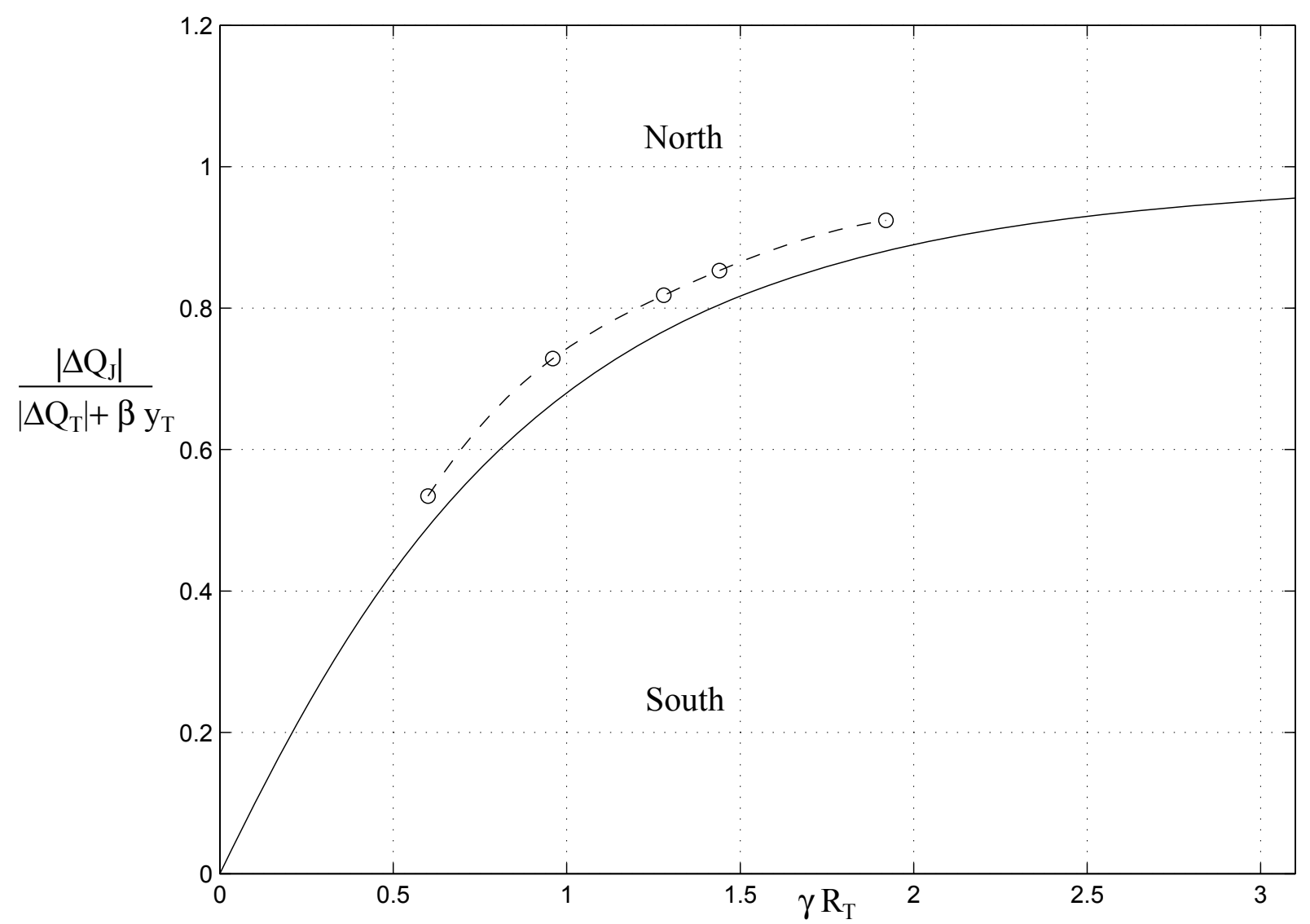

FIG.4e 

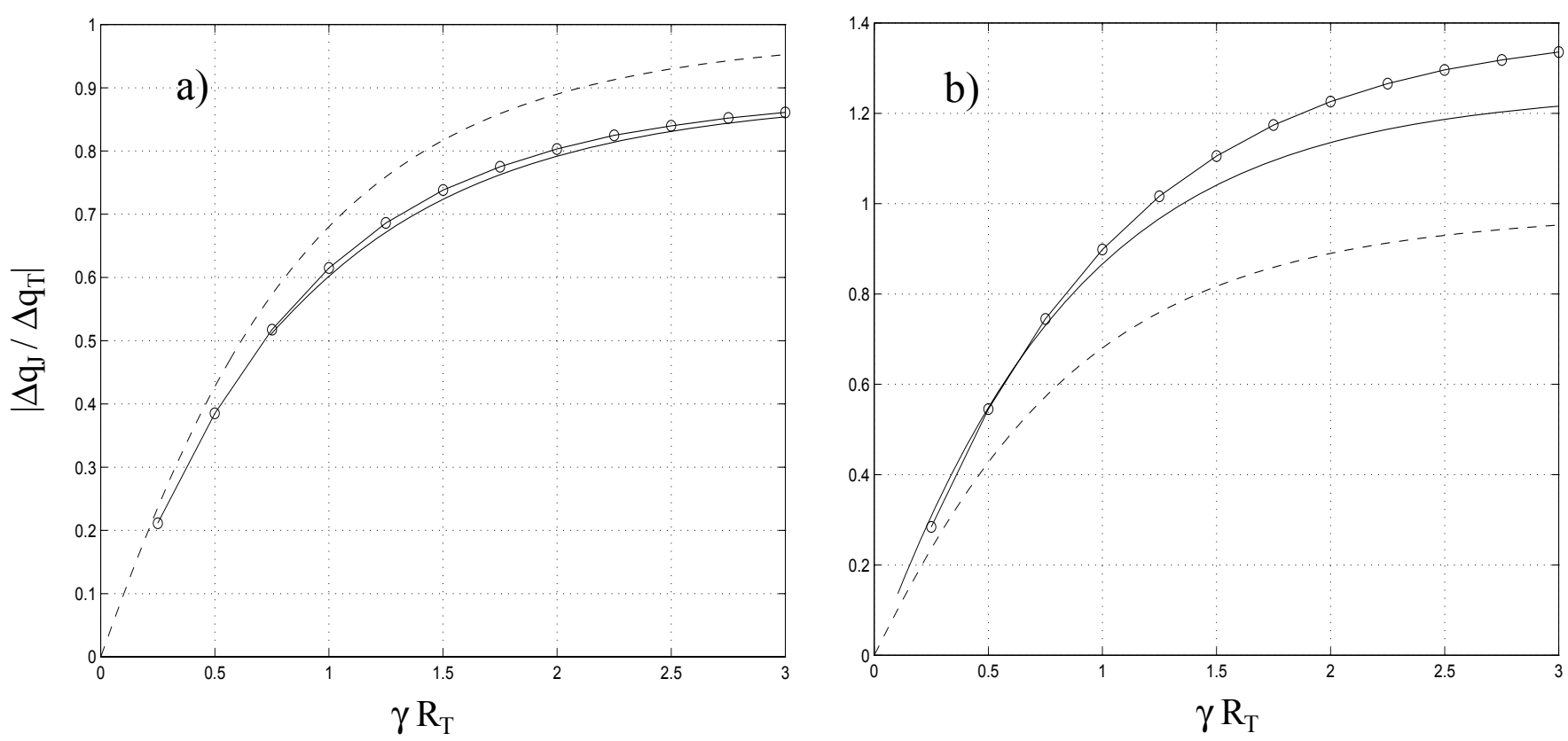

FIG.5ab 


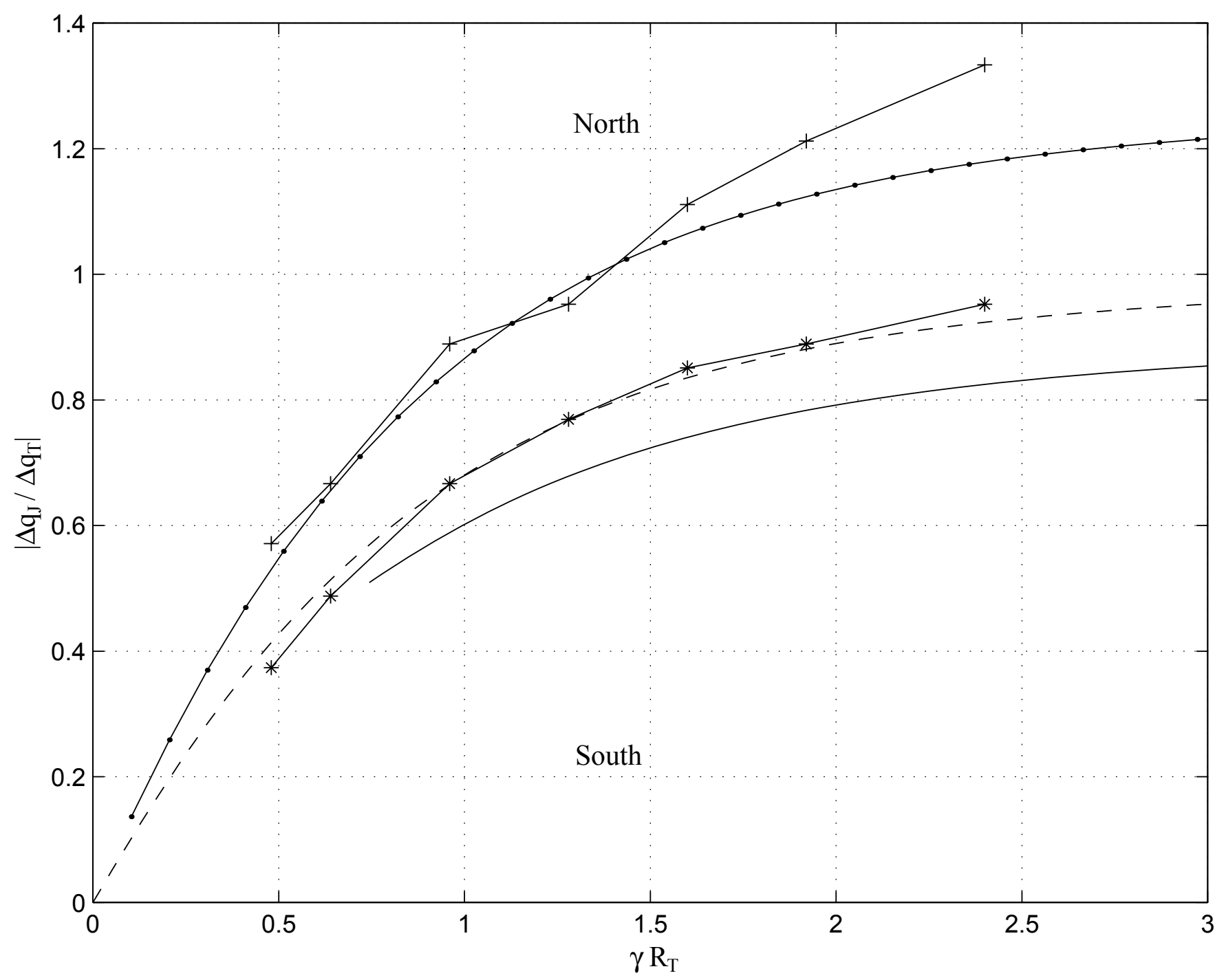

FIG.5c 
(5d)
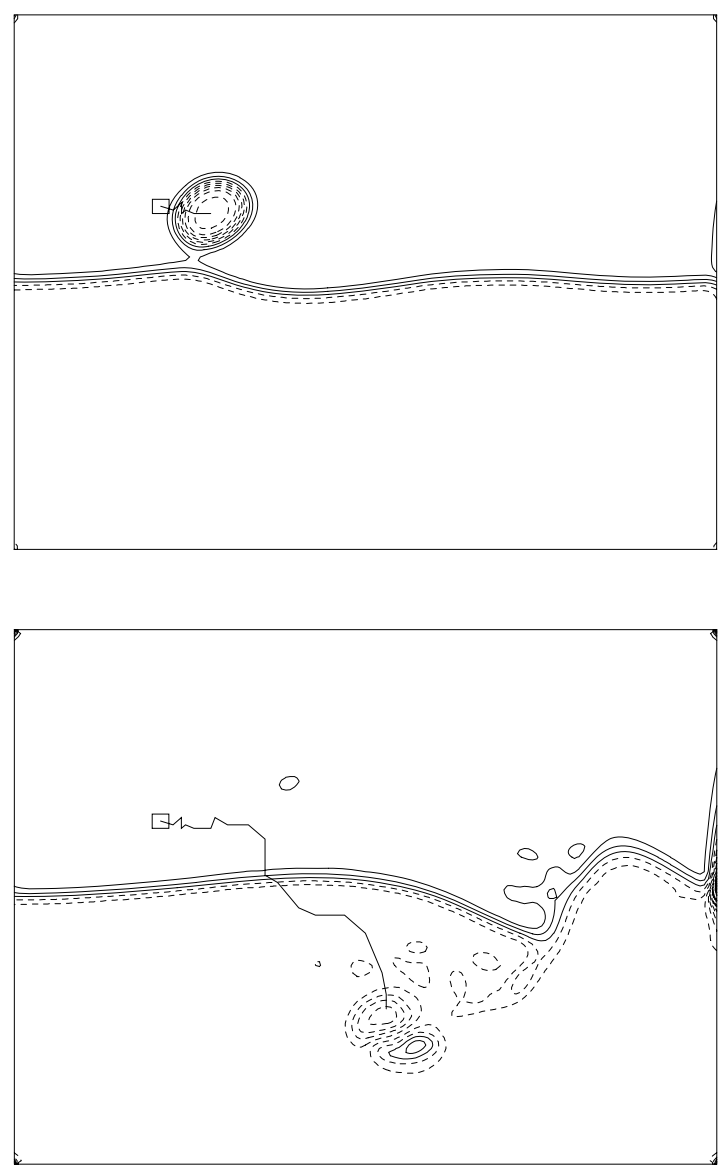

(5e)

$\mathrm{t}=130$
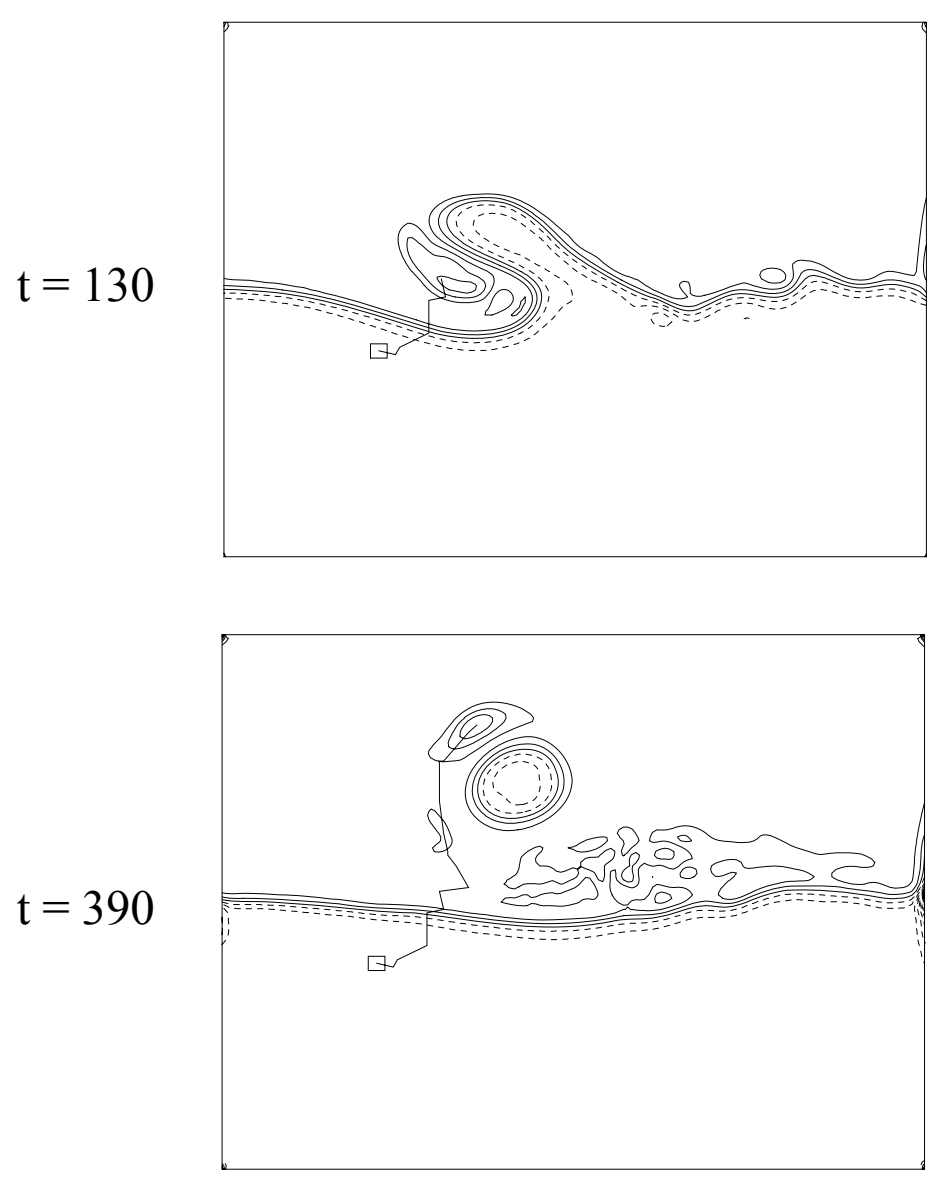

FIG.5de 
(5f)
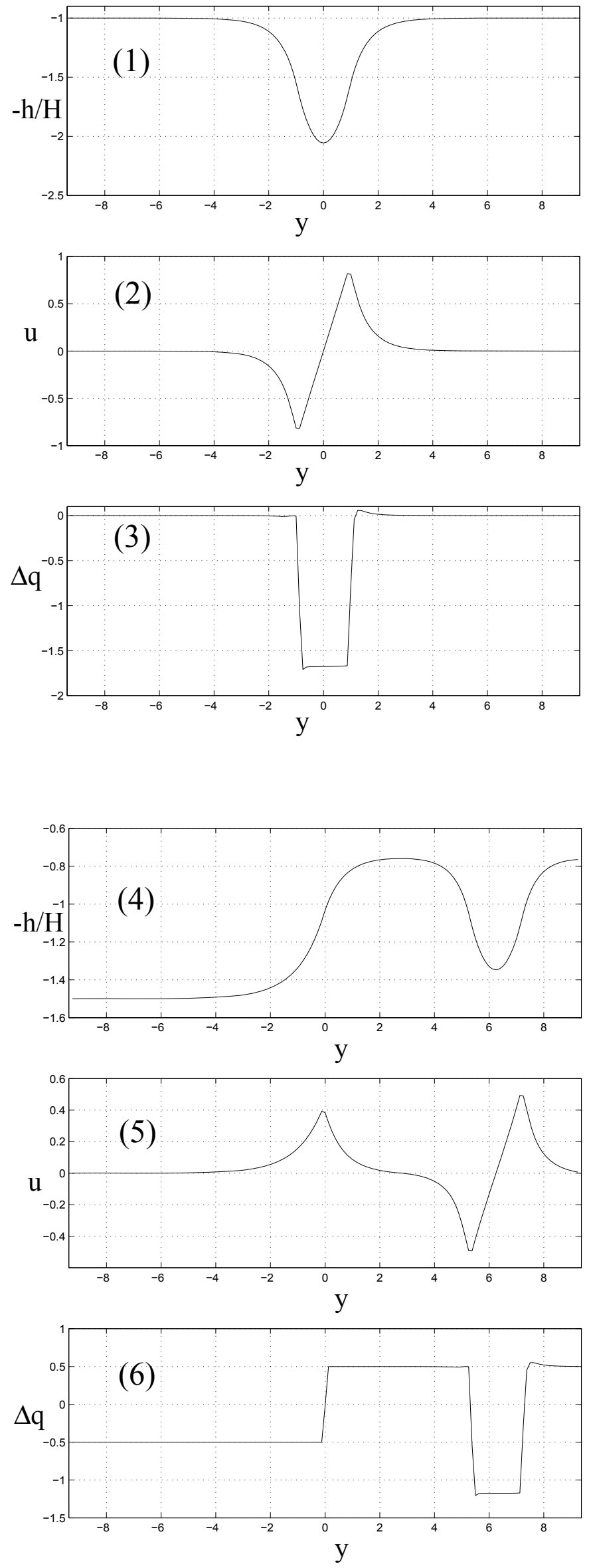

(5g)
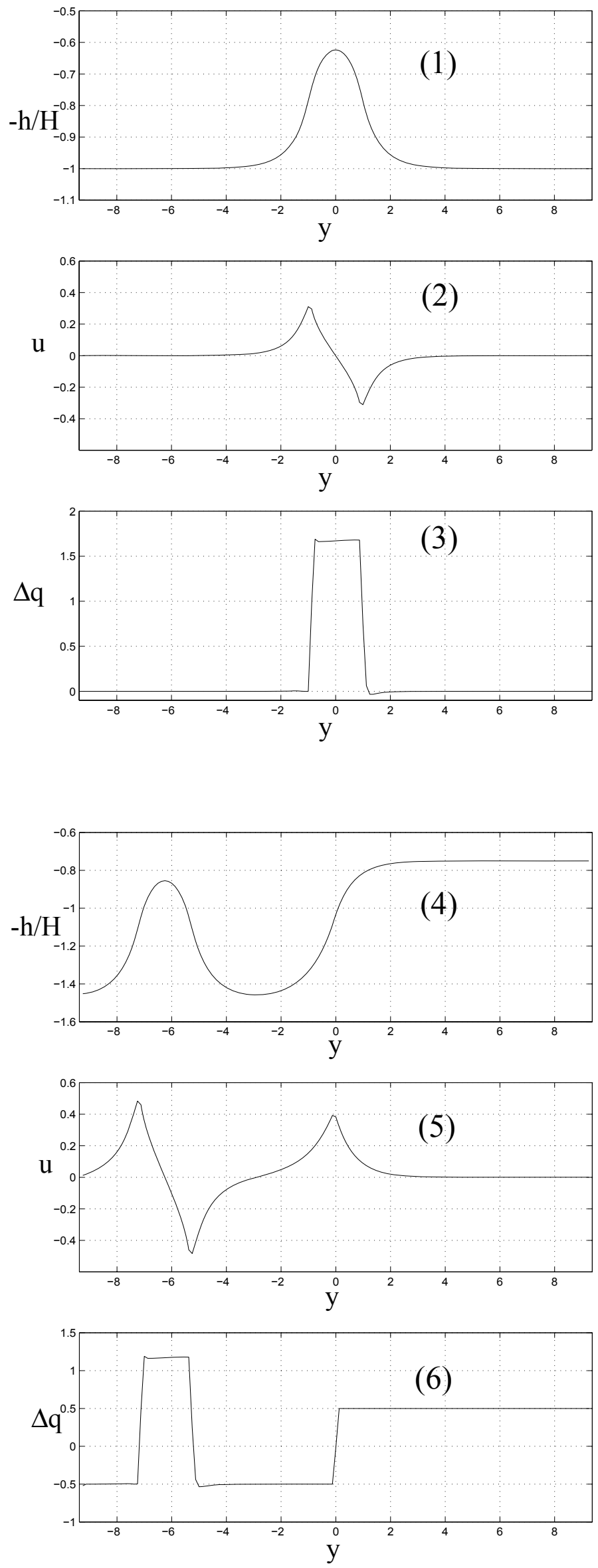

FIG.5fg 
(5h)
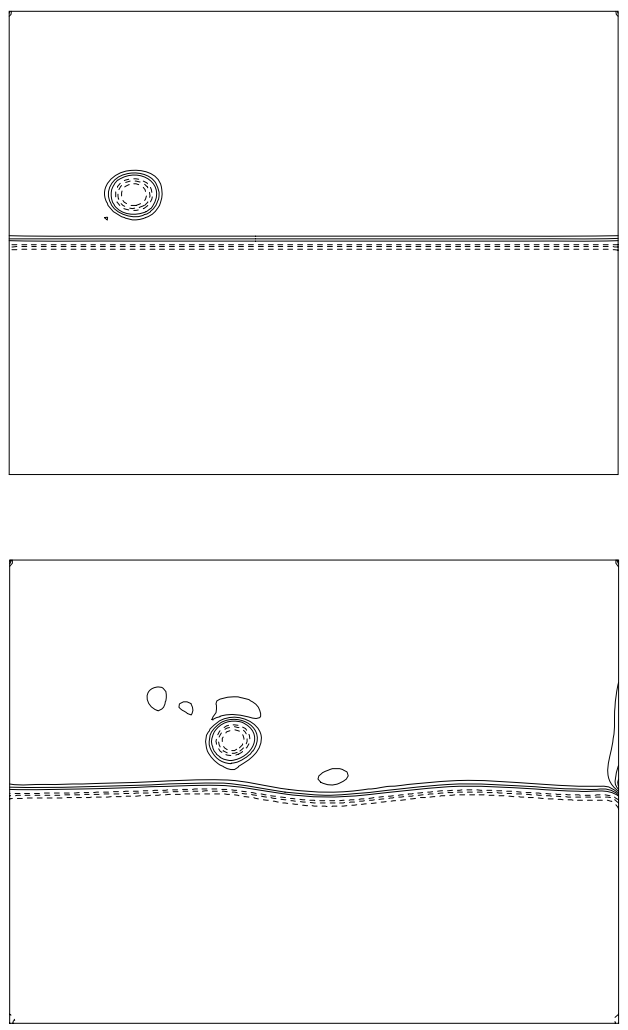

$\mathrm{t}=70$

$\mathrm{t}=0$
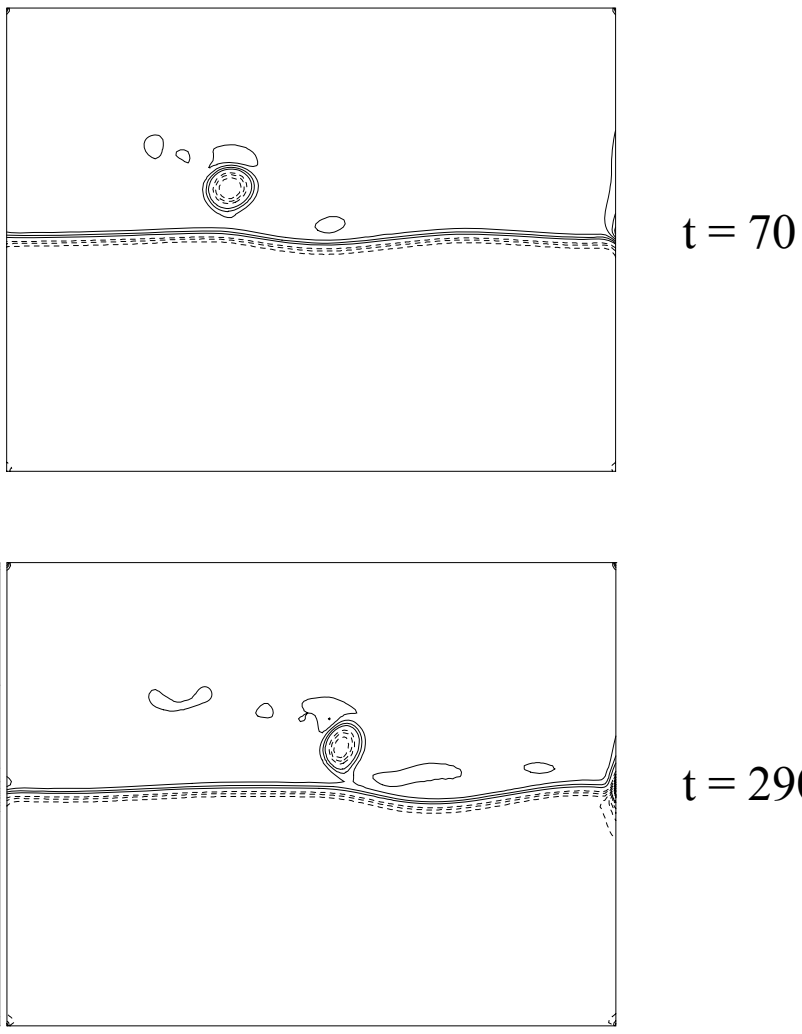

(5i)
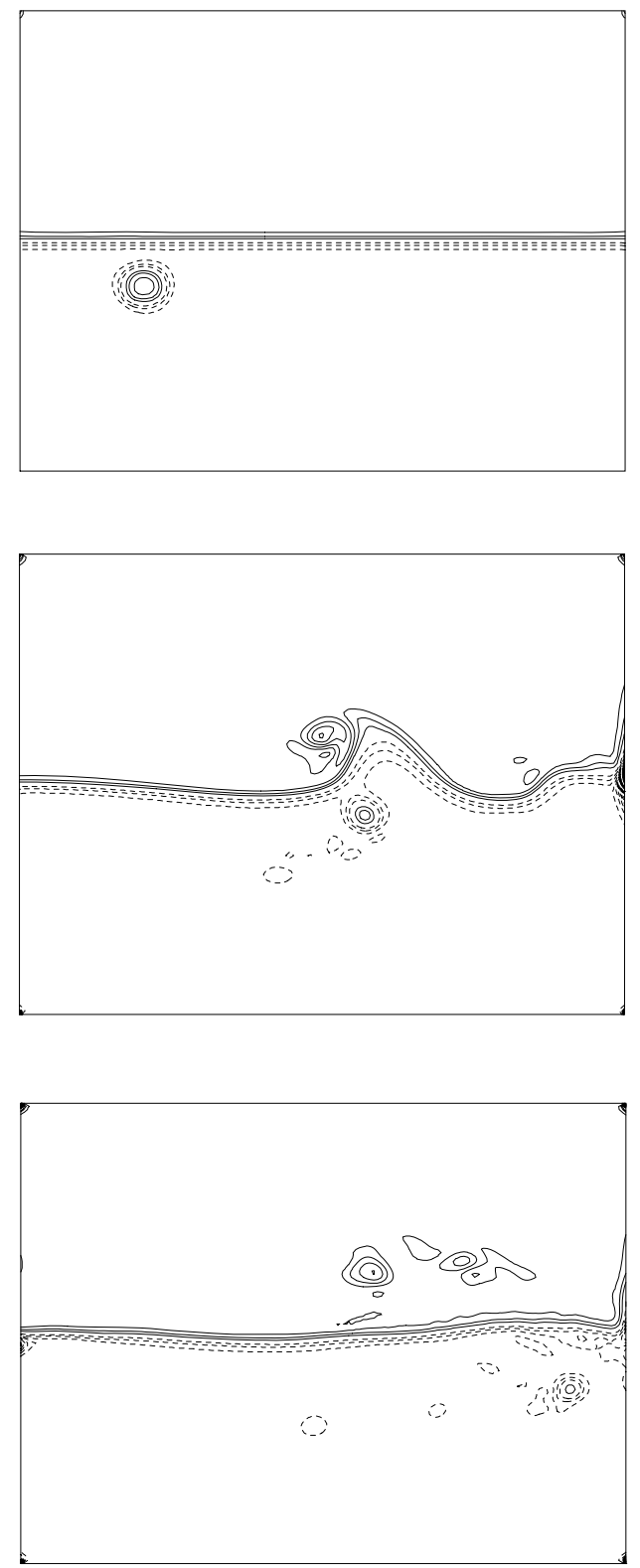

FIG.5hi 